\title{
SYMMETRIC INCLUSION PROCESS WITH SLOW BOUNDARY: HYDRODYNAMICS AND HYDROSTATICS
}

\author{
CHIARA FRANCESCHINI, PATRÍCIA GONÇALVES, AND FEDERICO SAU
}

\begin{abstract}
We study the hydrodynamic and hydrostatic limits of the one-dimensional open symmetric inclusion process with slow boundary. Depending on the value of the parameter tuning the interaction rate of the bulk of the system with the boundary, we obtain a linear heat equation with either Dirichlet, Robin or Neumann boundary conditions as hydrodynamic equation. In our approach, we combine duality and first-second class particle techniques to reduce the scaling limit of the inclusion process to the limiting behavior of a single, non-interacting, particle.
\end{abstract}

\section{INTRODUCTION}

Among the interacting particle systems employed to study non-equilibrium phenomena in mathematical statistical physics, the inclusion process is gaining increasing attention (see, e.g., 12, 48, 23, 7. 33). In particular, the symmetric inclusion process (SIP), introduced in 27 as discrete dual of a Gaussian energy process - known as Brownian momentum process (BMP) - and further studied in, e.g., 28, 12, can be considered as the "attractive" counterpart of the symmetric exclusion process (SEP). Indeed, inclusion particles evolve as independent random walks subject to an attractive rather than repulsive - interaction with nearest neighbors and, consequently, with no restriction on the maximal number of particles per site. Moreover, the SIP is related via some limiting "thermalization" procedures to the so-called KMP model [36], introduced as a microscopic model of heat transport in non-equilibrium.

The research around these stochastic systems mainly focuses on the microscopic structure of the non-equilibrium steady states as well as on their scaling limits, such as the derivation of Fick's law for the non-equilibrium steady state and the proof of local convergence to a Gibbs state, see, e.g., [36, 14. In this realm, as for the study of relaxation to the stationary non-equilibrium states, the first rigorous result on the derivation of the macroscopic equation governing the evolution of the density profile dates back to 20, 21. There the authors study the hydrodynamic and hydrostatic limits for gradient stochastic lattice gas models in a one-dimensional lattice. Further developments regarding scaling limits of such systems concern the study of - both dynamic and static - large deviations and non-equilibrium fluctuations around the hydrodynamic limit for the open SEP, see, e.g., [37, 22]. All these models yield parabolic equations with suitable Dirichlet boundary conditions as hydrodynamic equations.

More recently, stochastic models with more general interactions between the bulk of the system and the reservoirs have been introduced, see, e.g., 44, 29, 25, 30, 26, 16, 17, 18, 6]. Depending on the interaction chosen, more general boundary conditions - e.g., Robin, Neumann or nonlinear

2010 Mathematics Subject Classification. 60K35.

Key words and phrases. Symmetric inclusion process, hydrodynamic limit, hydrostatic limit, duality for Markov processes, heat equation with boundary conditions, first and second class particles.

Acknowledgments. F.S. wishes to thank Joe P. Chen for some fruitful discussions at an early stage of this work. C.F. and P.G. thank FCT/Portugal for support through the project UID/MAT/04459/2013. This project has received funding from the European Research Council (ERC) under the European Union's Horizon 2020 research and innovative programme (grant agreement No. 715734). F.S. thanks CAMGSD, IST, Lisbon, where part of this work has been done, and the European research and innovative programme No. 715734 for the kind hospitality. F.S. was founded by the European Union's Horizon 2020 research and innovation programme under the Marie-Skłodowska-Curie grant agreement No. 754411. 
boundary conditions - and more general nonlinear and fractional diffusions have been derived. For all these models, the so-called entropy and relative entropy methods (see, e.g., [35]) play a prominent role, but they both require replacement lemmas in order to close the equations at the microscopic level. Furthermore, in the context of exclusion processes in which a matrix formulation for the stationary non-equilibrium state is available and for zero-range processes in which the stationary non-equilibrium measures are of product form, explicit formulas for the stationary correlations simplify the study of hydrostatic and stationary non-equilibrium fluctuations.

For the open SIP, explicit expressions for the stationary correlations are not, in general, known. Furthermore, the entropy methods do not directly apply to this context because the partition function of the local Gibbs measures for SIP - products of Negative Binomial distributions - does not satisfy hypothesis [FEM] in [35] regarding their radius of convergence. Such an assumption, which is usually met for a wide class of zero-range and exclusion processes, is crucial in both oneand two-block estimates when applying entropy inequalities.

In view of the inapplicability of these general and robust methods, we base our study on the duality property of the open SIP. Duality in the context of interacting particle systems has been given a probabilistic "graphical" interpretation (see, e.g., [40]) and has been thoroughly explored from a Lie algebraic and generating function point of view (see, e.g., 28, 45, 11]), enriching both the class of models with the duality property and the space of duality functions for such models. In words, duality for a particle system may be viewed as the property of having suitably weighted factorial moments evolving according to a closed system of linear difference equations. Provided that $n \in \mathbb{N}$ is the degree of such moments, it turns out that the corresponding difference operators which govern their evolution is given by the infinitesimal generator of $n$ interacting particles which follow the same interaction rules as those of the original system. In the context of open systems in which particles enter and exit the bulk, the duality property still holds with the dual system having purely absorbing boundary. We remark that this picture is in line with its continuum counterpart, namely with the fact that stochastic representations of solutions of parabolic PDEs with Dirichlet boundary conditions are expressed in terms of diffusion processes which run backward in time and stop when hitting the boundary.

In this paper, we consider the open SIP on a one-dimensional lattice with nearest-neighbor interactions, whose boundary rates scale with the size of the system. As done in, e.g., 4, we introduce a parameter $\beta \geq 0$ which tunes the speed of these interactions: the higher the value of $\beta$, the slower the interaction. For this particle system, we derive the hydrodynamic and hydrostatic limits for all values of $\beta \geq 0$, obtaining, in particular, linear heat equations with either Dirichlet, Robin or Neumann boundary conditions depending on whether $\beta \in[0,1), \beta=1$ or $\beta \in(1, \infty)$.

Our strategy to derive the hydrodynamic limit may be summarized as follows. First we center our empirical density fields around their stationary part, which, in our case, is explicitly known. This centering procedure does not appear in previous literature on scaling limits of open systems and, by applying it also to the limiting fields, symmetry properties of continuum and discrete Laplacians become available and boundary terms in the limiting equation cancel out. Then, we exploit the linearity of the evolution equations for the first and second moments of the occupation variables to close the equations for the associated centered empirical density fields. To this purpose, a "corrected empirical density field" argument (see, e.g., 32]) and the centering with respect to the stationary part are crucial in order to close the equations for the fields and avoid technical replacement lemmas - based, ultimately, on relative entropy estimates - as done in the context of the open SEP in, e.g., 4, 29.

For what concerns the hydrostatic limit, in order to verify that the stationary non-equilibrium measures satisfy the assumptions of the hydrodynamic limit, in general, one needs to control the stationary two-point correlations. This has been done for the slow-boundary SEP in [4] (see also [37]) by using the explicit form of such correlations, and in [47. (see also 38]) by proving replacement lemmas near the boundary. As already mentioned, for SIP, matrix formulations of the non-equilibrium steady state, explicit formulas for the correlations and replacement lemmas are not available. In 
order to overcome this, we develop a self-contained method - which is one of the main contributions of this work - to derive hydrostatic limits based solely on duality and a hierarchical representation of the dual particle system. More specifically, we express correlations in terms of a dual system of two inclusion particles as in 23], and combine this with the introduction of a hierarchical "first-second class particle" construction for SIP. This allows us to reduce the problem of checking the $L^{1}$-decay of these two-point stationary correlations to the study of a single one-dimensional random walk with absorbing boundary, considerably simplifying the analysis.

Duality techniques are not new in the context of scaling limits for non-equilibrium systems and have been used thoroughly - even without explicit mention (see, e.g., [37, 4, 16]). In this paper, we show that first and second order dualities combined with a purely probabilistic "lookdown" construction of the dual system provide a simple strategy to obtain both hydrodynamic and hydrostatic limit, avoiding both non-homogeneous evolution equations for the two point correlations and replacement lemmas as, e.g., in [4].

We emphasize that the duality property has to be considered an "exact" feature. Indeed, although some notions of approximate duality proved to be useful in some perturbative contexts (see, e.g., [13, Chapter 6]), in general, duality does not transfer to perturbations of the particle systems, which, for instance, introduce asymmetries. Nevertheless, the duality property is robust with respect to the generalizations of the underlying geometries, as, e.g., with respect to the introduction of disorder or the dimension of the lattice, as well as, of the reservoir interaction (see, e.g., 23). Moreover, as for the stationary two-point correlations, the aforementioned reduction from two to one dual particles is general and holds for all geometries and reservoir interactions, even when the stationary particle density profile is not explicitly known. In this sense, we believe our techniques to apply to a larger family of discrete and continuum open systems for which analogous duality relations (see [12]) as well as hierarchical constructions for the dual processes hold. Among these models, we mention the open symmetric exclusion process $\operatorname{SEP}(\alpha)$, where up to $\alpha \in \mathbb{N}$ particles are allowed to each site (see, e.g., [12, whose jump rates differ from those of the non-gradient generalized exclusion process studied, e.g., in [35]) and the continuum BEP and KMP models.

We further observe that, by following our approach, the regime of fast boundary - corresponding to $\beta<0$, still remains open because a control uniform in time on the total mass of particles in the bulk is not at hand for the open SIP as in the case, e.g., of SEP and zero-range processes. Indeed, the total mass of the system can be uniformly dominated due to hard core constraints for the open SEP and due to the monotonicity - or, attractiveness - of the zero-range process (see, e.g., [26]), property which is not satisfied by SIP. A second challenge consists in the study of nonequilibrium and stationary fluctuations as well as of dynamic and static large deviations around the hydrodynamic and hydrostatic limits, respectively, for the open SIP. This is left for a future work.

We conclude this introduction with a short outline on the organization of the paper. In Section 2 we introduce the particle system, the associated equilibrium and non-equilibrium measures, the dual process and the duality relations. Moreover, we define the functional setting we use to describe our main results, the hydrodynamic and hydrostatic limits stated in Section 3 . Section 4 is devoted to the proofs of the two main results. We conclude the paper with two appendices. In Appendix A we present a complete and unified construction of the function spaces used for which existence and uniqueness of the solution to the hydrodynamic equations we consider follows at once. In Appendix $\mathrm{B}$, we prove a result for a one-dimensional random walk required in the proof of the hydrostatic limit.

\section{Setting}

In this section, we introduce the particle system in contact with reservoirs, the duality properties and its stationary measures. Then, we present the function spaces and the weak formulation of the limiting hydrodynamic equations. 
2.1. Open symmetric inclusion process. Let $N \in \mathbb{N}$ play the role of scaling parameter and $\Lambda_{N}:=\{1, \ldots, N-1\}$ be the one-dimensional chain on which the particles hop. We define by $\mathcal{X}_{N}$ the configuration space given by $\mathcal{X}_{N}:=\mathbb{N}_{0}^{\Lambda_{N}}=\{0,1, \ldots\}^{\Lambda_{N}}$, where, for any given $\eta \in \mathcal{X}_{N}$ and $x \in \Lambda_{N}, \eta(x)$ stands for the number of particles of the configuration $\eta$ at site $x$, referred to as occupation variable at $x$. The stochastic dynamics is described by the infinitesimal generator $\mathcal{L}^{N}$ whose action on local functions $f: \mathcal{X}_{N} \rightarrow \mathbb{R}$ is given by

$$
\mathcal{L}^{N} f:=\mathcal{L}_{\text {bulk }}^{N} f+\mathcal{L}_{L}^{N} f+\mathcal{L}_{R}^{N} f,
$$

where

$$
\begin{aligned}
\mathcal{L}_{\text {bulk }}^{N} f(\eta) & :=N^{2} \sum_{x \in \Lambda_{N} \backslash\{N-1\}}\left\{\begin{array}{r}
\eta(x)(\alpha+\eta(x+1))\left(f\left(\eta^{x, x+1}\right)-f(\eta)\right) \\
+\eta(x+1)(\alpha+\eta(x))\left(f\left(\eta^{x+1, x}\right)-f(\eta)\right)
\end{array}\right\}, \\
\mathcal{L}_{L}^{N} f(\eta) & :=N^{2-\beta}\left\{\begin{array}{r}
\alpha_{L} \vartheta_{L}(\alpha+\eta(1))\left(f\left(\eta^{1,+}\right)-f(\eta)\right) \\
+\eta(1)\left(\alpha_{L}+\alpha_{L} \vartheta_{L}\right)\left(f\left(\eta^{1,-}\right)-f(\eta)\right)
\end{array}\right\}
\end{aligned}
$$

and

$$
\mathcal{L}_{R}^{N} f(\eta):=N^{2-\beta}\left\{\begin{array}{r}
\alpha_{R} \vartheta_{R}(\alpha+\eta(N-1))\left(f\left(\eta^{N-1,+}\right)-f(\eta)\right) \\
+\eta(N-1)\left(\alpha_{R}+\alpha_{R} \vartheta_{R}\right)\left(f\left(\eta^{N-1,-}\right)-f(\eta)\right)
\end{array}\right\} .
$$

In the above expressions, $\eta^{x, y}$ stands for the configuration obtained from $\eta$ by removing a particle from site $x \in \Lambda_{N}$ (if any) and placing it at site $y \in \Lambda_{N}$, i.e., $\eta^{x, y}:=\eta-\delta_{x}+\delta_{y} \in \mathcal{X}_{N}$ with $\delta_{x}$ denoting the configuration consisting of a single particle at site $x \in \Lambda_{N}$. Furthermore,

$$
\begin{array}{ll}
\eta^{1,+}:=\eta+\delta_{1} & \eta^{N-1,+}:=\eta+\delta_{N-1} \\
\eta^{1,-}:=\eta-\delta_{1} & \eta^{N-1,-}:=\eta-\delta_{N-1} .
\end{array}
$$

The parameters $\alpha, \alpha_{L}, \alpha_{R}, \vartheta_{L}$ and $\vartheta_{R}$ are all positive and, while $\alpha$ stands for the bulk site attraction parameter, the others describe the interaction of the system with left and right reservoirs through the ending sites of the chain $\Lambda_{N}$. We remark that interpreting $\alpha_{L}$ and $\alpha_{R}$, resp.

$$
\rho_{L}:=\alpha_{L} \vartheta_{L} \quad \text { and } \quad \rho_{R}:=\alpha_{R} \vartheta_{R},
$$

as the reservoirs' attraction, resp. reservoirs' particle density, parameters, the jump rates due to the reservoir interaction have exactly the same form as the jump rates in the bulk. Lastly, the parameter $\beta \geq 0$ tunes the intensity of the reservoir interaction (see also Figure 1 below). We warn the reader that above and in what follows, for notational convenience, the dependence on $\beta \geq 0$ is never explicitly mentioned.

In what follows, for all $\mu$ probability measures on $\mathcal{X}_{N}$, we let $\mathbb{P}_{\mu}^{N}$ and $\mathbb{E}_{\mu}^{N}$ denote the probability law and corresponding expectation of the process with generator $\mathcal{L}^{N}$ in (2.1) with initial distribution given by $\mu$. If the initial distribution is a Dirac measure, we will adopt the following shortcut: for all $\eta \in \mathcal{X}_{N}, \mathbb{P}_{\eta}^{N}:=\mathbb{P}_{\delta_{\eta}}^{N}$ and $\mathbb{E}_{\eta}^{N}:=\mathbb{E}_{\delta_{\eta}}^{N}$.

There is an immediate comparison of the open inclusion dynamics with the corresponding open exclusion dynamics: with the additional requirement of setting $\alpha \in \mathbb{N}$, the exclusion dynamics in the bulk is recovered by replacing the plus sign in the jump rates with the negative sign, e.g., $\eta(x)(\alpha-\eta(x+1))$ in place of $\eta(x)(\alpha+\eta(x+1))$; similarly for what concerns the reservoir interaction with the further restriction $\vartheta_{L}, \vartheta_{R} \in(0,1)$.

Remark 2.1 (NOTATION). An alternative parametrization (see, e.g., 12, 29] for the SEP) of the open SIP employs positive parameters $a_{L}, a_{R}, b_{L}$ and $b_{R}$ as follows:

$$
\mathcal{L}_{L}^{N} f(\eta):=N^{2-\beta}\left\{\begin{array}{r}
b_{L}(\alpha+\eta(1))\left(f\left(\eta^{1,+}\right)-f(\eta)\right) \\
+a_{L} \eta(1)\left(f\left(\eta^{1,-}\right)-f(\eta)\right)
\end{array}\right\}
$$



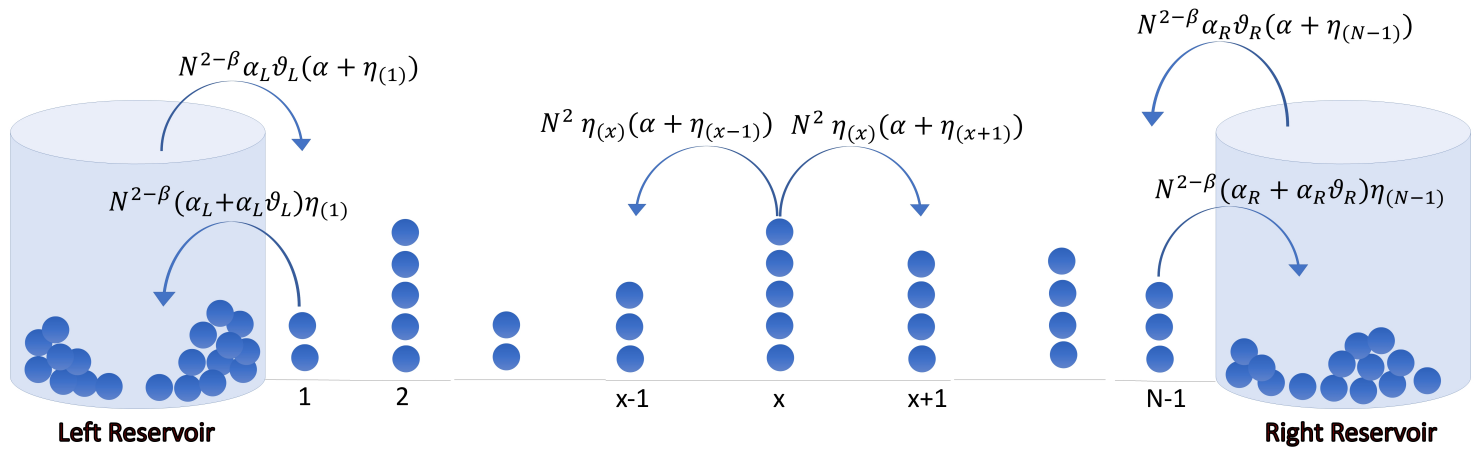

Figure 1. Schematic description of the dynamics of the one-dimensional open SIP on $\Lambda_{N}$ with parameters $\alpha, \alpha_{L}, \alpha_{R}, \vartheta_{L}, \vartheta_{R}>0$ and $\beta \geq 0$.

and

$$
\mathcal{L}_{R}^{N} f(\eta):=N^{2-\beta}\left\{\begin{array}{r}
b_{R}(\alpha+\eta(N-1))\left(f\left(\eta^{N-1,+}\right)-f(\eta)\right) \\
+a_{R} \eta(N-1)\left(f\left(\eta^{N-1,+}\right)-f(\eta)\right)
\end{array}\right\},
$$

which corresponds to setting

$$
\begin{array}{ll}
a_{L}=\alpha_{L}\left(1+\vartheta_{L}\right) & b_{L}=\alpha_{L} \vartheta_{L} \\
a_{R}=\alpha_{R}\left(1+\vartheta_{R}\right) & b_{R}=\alpha_{R} \vartheta_{L} .
\end{array}
$$

The bulk dynamics for the open SIP is conservative and the total number of particles changes only due to particle injection and absorption of the reservoirs. Unlike the exclusion process for which each site may be occupied by at most a finite number of particles, for the inclusion process the occupation variables admit no prescribed upper bound. Nevertheless, in view of the form of the boundary interaction rates and classical results on birth-death processes, the particle system does not explode, ensuring its existence for any finite initial configuration and any time.

Proposition 2.1 (NON-EXPlosiveness). For all $N \in \mathbb{N}, \beta \geq 0$ and initial configurations $\eta \in \mathcal{X}_{N}$, the open SIP $\left\{\eta_{t}^{N}: t \geq 0\right\}$ with generator $\mathcal{L}^{N}$ is non-explosive; namely, almost surely, in any bounded interval of time the system undergoes finitely many transitions and

$$
\left\|\eta_{t}^{N}\right\|_{\ell_{N}^{1}}:=\sum_{x \in \Lambda_{N}} \eta_{t}^{N}(x)<\infty, \quad t \geq 0
$$

Proof. The stochastic process

$$
\left\{\left\|\eta_{t}^{N}\right\|_{\ell_{N}^{1}}: t \geq 0\right\}
$$

on $\mathbb{N}_{0}$ is stochastically dominated by the pure birth process on $\mathbb{N}_{0}$ started from $\left\|\eta_{0}^{N}\right\|_{\ell_{N}^{1}}=\|\eta\|_{\ell_{N}^{1}}$ and with birth rates $\left\{r_{n}: n \in \mathbb{N}_{0}\right\}$ given by

$$
r_{n}:=N^{2-\beta}\left(\alpha_{L} \vartheta_{L}+\alpha_{R} \vartheta_{R}\right)(\alpha+n), \quad n \in \mathbb{N}_{0} .
$$


Since $\sum_{n \in \mathbb{N}_{0}} \frac{1}{r_{n}}=\infty$, such birth process is non-explosive and, thus, by stochastic domination, also the process in 2.3 .

2.1.1. Stationary equilibrium and non-equilibrium measures. In absence of reservoirs, the SIP admits a one-parameter family of reversible product measures with marginals given by Negative Binomials with shape parameter $\alpha>0$ (see, e.g., [28, 12]):

$$
\left\{\mu_{\vartheta}^{N}:=\otimes_{x \in \Lambda_{N}} \nu_{\vartheta}: \vartheta>0\right\} \quad \text { with } \quad \nu_{\vartheta} \sim \operatorname{NegBin}\left(\alpha, \frac{\vartheta}{1+\vartheta}\right)
$$

where our parametrization of $\nu_{\vartheta}$ is such that, for all $x \in \Lambda_{N}$,

$$
E_{\mu_{\vartheta}^{N}}[\eta(x)]=\vartheta \alpha \quad \text { and } \quad E_{\mu_{\vartheta}^{N}}\left[(\eta(x)-\alpha \vartheta)^{2}\right]=\vartheta(1+\vartheta) \alpha .
$$

Here and in the sequel, for all $\mu$ probability measures, $E_{\mu}$ denotes expectation with respect to $\mu$. In presence of reservoirs, there exists a unique stationary measure $\mu_{\vartheta_{L}, \vartheta_{R}}^{N}$ and, depending on the values of $\vartheta_{L}$ and $\vartheta_{R}$, two different scenarios occur (see, e.g., [12, 23] for more details and proofs): if $\vartheta_{L}=\vartheta_{R}=\vartheta>0$, the system is in equilibrium and the unique stationary - actually reversible measure $\mu_{\vartheta_{L}, \vartheta_{R}}^{N}$ is given by $\mu_{\vartheta}^{N}$ in 2.4 , thus, is product and independent of the parameters $\beta, \alpha_{L}$ and $\alpha_{R}$. If $\vartheta_{L} \neq \vartheta_{R}$, the system is out of equilibrium and the unique stationary measure is not in product form, does depend on $\beta, \alpha_{L}, \alpha_{R}$ and it is only partially characterized (see [23]). Indeed, no matrix formulation as for the open exclusion (see, e.g., [14]) is available for the inclusion process and, hence, two-point (and higher order) correlations are not, in general, explicit. However, to the purpose of deriving the hydrostatic limit for the open inclusion process, the partial characterization provided in 23] plays a crucial role.

2.2. Duality. The duality property will be a key ingredient for all our results. In words, duality for a pair of Markov processes consists in finding an observable - the so-called duality function - of the joint system whose expectation with respect to the evolution of one marginal equals the expectation with respect to the evolution of the second marginal. In the context of interacting particle systems, duality typically relates the expectation of suitable $n$-joint moments of the occupation variables of one system with the evolution of $n$ dual interacting particles. Moreover, in presence of reservoirs, duality relates open systems to dual particle systems with purely absorbing boundary. Such a correspondence is related to the well-known Feynman-Kac formulas for parabolic solutions to PDEs with boundary conditions (see, e.g., [43. Chapter 9]), where, in the context of interacting particle systems, this Feynman-Kac formula holds not only for the expected density of particles, but also for suitable higher order moments.

2.2.1. Absorbing process and duality relation. Before introducing the duality function, let us describe the absorbing symmetric inclusion process, dual to the process with generator $\mathcal{L}^{N}$ defined in 2.1 . For such a dual process, particles evolve on the extended lattice $\widehat{\Lambda}_{N}:=\{0,1, \ldots, N\}$ and we let $\widehat{\mathcal{X}}_{N}:=\mathbb{N}_{0}^{\widehat{\Lambda}_{N}}$ denote the dual configuration space. The infinitesimal generator of the dual process, $\widehat{\mathcal{L}}^{N}$, is given, for all local functions $f: \widehat{\mathcal{X}}_{N} \rightarrow \mathbb{R}$, by

$$
\widehat{\mathcal{L}}^{N} f:=\widehat{\mathcal{L}}_{\text {bulk }}^{N} f+\widehat{\mathcal{L}}_{L}^{N} f+\widehat{\mathcal{L}}_{R}^{N} f,
$$

where the bulk dynamics coincides with that of the open SIP, namely, for all $\xi \in \widehat{\mathcal{X}}$,

$$
\widehat{\mathcal{L}}_{\text {bulk }}^{N} f(\xi):=N^{2} \sum_{x \in \Lambda_{N} \backslash\{N-1\}}\left\{\begin{array}{r}
\xi(x)(\alpha+\xi(x+1))\left(f\left(\xi^{x, x+1}\right)-f(\xi)\right) \\
+\xi(x+1)(\alpha+\xi(x))\left(f\left(\xi^{x+1, x}\right)-f(\xi)\right)
\end{array}\right\},
$$

while the dynamics at left and right ends of $\widehat{\Lambda}_{N}$ is purely absorbing: for all $\xi \in \widehat{\mathcal{X}}_{N}$,

$$
\begin{aligned}
& \widehat{\mathcal{L}}_{L}^{N} f(\xi):=N^{2-\beta} \alpha_{L} \xi(1)\left(f\left(\xi^{1,0}\right)-f(\xi)\right) \\
& \widehat{\mathcal{L}}_{R}^{N} f(\xi):=N^{2-\beta} \alpha_{R} \xi(N-1)\left(f\left(\xi^{N-1, N}\right)-f(\xi)\right) .
\end{aligned}
$$


We observe that this stochastic dynamics conserves the total number of particles in the system. Moreover, for all $\widehat{\mu}$ probability measures on $\widehat{\mathcal{X}}_{N}$, we let $\widehat{\mathbb{P}}_{\widehat{\mu}}^{N}$ and $\widehat{\mathbb{E}}_{\widehat{\mu}}^{N}$ denote the probability law and corresponding expectation of the process with generator $\widehat{\mathcal{L}}^{N}$ in 2.5 with initial distribution given by $\widehat{\mu}$. For notational convenience, for all $\xi \in \widehat{\mathcal{X}}_{N}, \widehat{\mathbb{P}}_{\xi}^{N}:=\widehat{\mathbb{P}}_{\delta_{\xi}}^{N}$ and $\widehat{\mathbb{E}}_{\xi}^{N}:=\widehat{\mathbb{E}}_{\delta_{\xi}}^{N}$.

Let us define the following function $\mathcal{D}_{N}: \widehat{\mathcal{X}} \times \mathcal{X} \rightarrow \mathbb{R}$ given by

$$
\mathcal{D}_{N}(\xi, \eta):=\left(\vartheta_{L}\right)^{\xi(0)}\left(\prod_{x \in \Lambda_{N}} d(\xi(x), \eta(x))\right)\left(\vartheta_{R}\right)^{\xi(N)},
$$

where

We remark that, for all $n \in \mathbb{N}_{0}$,

$$
d(k, n):=\frac{n !}{(n-k) !} \frac{\Gamma(\alpha)}{\Gamma(\alpha+k)} \mathbf{1}_{\{k \leq n\}}, \quad k, n \in \mathbb{N}_{0} .
$$

$$
d(0, n)=1, \quad d(1, n)=\frac{n}{\alpha} \quad \text { and } \quad d(2, n)=\frac{n(n-1)}{\alpha(\alpha+1)},
$$

and, more generally, $d(k, n)$ is a weighted $k$-th falling factorial for the $n$-variable. Moreover, we will need the following property concerning factorial moments of Negative Binomial distributions: for all $k \in \mathbb{N}_{0}$ and $\vartheta>0$,

$$
E_{\nu_{\vartheta}}[d(k, \cdot)]=\sum_{n \in \mathbb{N}_{0}} d(k, n) \nu_{\vartheta}(n)=\vartheta^{k} .
$$

It was shown in [12] that the open and absorbing SIP are dual with the function $\mathcal{D}_{N}$ in 2.6 as duality function, i.e., the following identity - referred to as duality relation,

$$
\mathcal{L}^{N} \mathcal{D}_{N}(\xi, \cdot)(\eta)=\widehat{\mathcal{L}}^{N} \mathcal{D}_{N}(\cdot, \eta)(\xi)
$$

holds for all $N \in \mathbb{N}, \beta \geq 0, \eta \in \mathcal{X}_{N}$ and $\xi \in \widehat{\mathcal{X}}_{N}$. We note that the dual system stochastic dynamics does not depend on the parameters $\vartheta_{L}$ and $\vartheta_{R}$, while the duality function $\mathcal{D}_{N}$ does. By Kolmogorov equations, the infinitesimal relation $(2.8)$ establishes that, for all $\eta \in \mathcal{X}_{N}$, the function

$$
(t, \xi) \mapsto \mathbb{E}_{\eta}^{N}\left[\mathcal{D}_{N}\left(\xi, \eta_{t}\right)\right]
$$

is the solution of the following deterministic linear Cauchy problem:

$$
\left\{\begin{array}{rlrl}
\frac{d}{d t} f(t, \xi) & =\widehat{\mathcal{L}}^{N} f(t, \xi) & , \quad \xi \in \widehat{\mathcal{X}}_{N}, t \geq 0 \\
f(0, \xi) & =\mathcal{D}_{N}(\xi, \eta) \quad, \quad \xi \in \widehat{\mathcal{X}}_{N} .
\end{array}\right.
$$

2.2.2. One and two dual particles. For the sequel, it will be important to express the duality relation in 2.8 and its consequences in terms of labeled dual particles. The two cases of interest are those in which the dual system consists of either one or two particles only.

For what concerns the case of just one particle, (2.8) rewrites as

$$
\mathcal{L}^{N} D_{N}(x, \cdot)(\eta)=A^{N} D_{N}(\cdot, \eta)(x)
$$

where, for all $\eta \in \mathcal{X}_{N}$,

$$
D_{N}(x, \eta):=\mathcal{D}_{N}\left(\delta_{x}, \eta\right)= \begin{cases}\frac{\eta(x)}{\alpha} & \text { if } x \in \Lambda_{N} \\ \vartheta_{L} & \text { if } x=0 \\ \vartheta_{R} & \text { if } x=N\end{cases}
$$

and $A^{N}$ is the generator of a single - thus, non-interacting - particle on $\widehat{\Lambda}_{N}$ with the two endpoints $\{0, N\}$ being absorbing: for all $f: \widehat{\Lambda}_{N} \rightarrow \mathbb{R}$,

$$
\begin{aligned}
A^{N} f(x) & :=\mathbf{1}_{\left\{x \in \Lambda_{N}\right\}} N^{2} \sum_{y \in \Lambda_{N}} \mathbf{1}_{\{|y-x|=1\}} \alpha(f(y)-f(x)) \\
& +\mathbf{1}_{\{x=1\}} N^{2-\beta} \alpha_{L}(f(0)-f(1))
\end{aligned}
$$




$$
+\mathbf{1}_{\{x=N-1\}} N^{2-\beta} \alpha_{R}(f(N)-f(N-1)) .
$$

Let us observe that, restricted to the subspace of functions $f: \widehat{\Lambda}_{N} \rightarrow \mathbb{R}$ which equal zero at the boundary $\{0, N\}, A^{N}$ is symmetric, i.e., for all $f, g: \widehat{\Lambda}_{N} \rightarrow \mathbb{R}$ such that $f(0)=f(N)=g(0)=$ $g(N)=0$, we have

$$
\left\langle\left\langle f, A^{N} g\right\rangle_{N}=\left\langle\left\langle A^{N} f, g\right\rangle_{N}\right.\right.
$$

where

$$
\left\langle\langle f, g\rangle_{N}:=\frac{1}{N} \sum_{x \in \Lambda_{N}} f(x) g(x) \alpha .\right.
$$

Regarding the case of a dual system consisting of two particles, analogous considerations hold and $(2.8)$ boils down to

$$
\mathcal{L}^{N} D_{N}(x, y, \cdot)(\eta)=B^{N} D_{N}(\cdot, \cdot, \eta)(x, y)
$$

where, for all $\eta \in \mathcal{X}_{N}$,

$$
D_{N}(x, y, \eta):=\mathcal{D}_{N}\left(\delta_{x}+\delta_{y}, \eta\right)= \begin{cases}\frac{\eta(x)(\eta(x)-1)}{\alpha(\alpha+1)} & \text { if } x=y \in \Lambda_{N} \\ D_{N}(x, \eta) D_{N}(y, \eta) & \text { otherwise }\end{cases}
$$

and $B^{N}$ is the generator of two inclusion particles on $\widehat{\Lambda}_{N}$ with absorbing sites $\{0, N\}$ :

$$
\begin{aligned}
B^{N} f(x, y): & =A^{N} f(\cdot, y)(x)+A^{N} f(x, \cdot)(y) \\
& +N^{2} \mathbf{1}_{\{x, y \neq 0, N\}} \mathbf{1}_{\{|x-y|=1\}}((f(x, x)-f(x, y))+(f(y, y)-f(x, y))),
\end{aligned}
$$

for all functions $f: \widehat{\Lambda}_{N} \times \widehat{\Lambda}_{N} \rightarrow \mathbb{R}$. For such functions, let us introduce the following inner product

$$
\langle\langle f, g\rangle\rangle_{N \times N}:=\frac{1}{N^{2}} \sum_{x \in \Lambda_{N}} \sum_{y \in \Lambda_{N}} f(x, y) g(x, y) \alpha\left(\alpha+\mathbf{1}_{\{x=y\}}\right) .
$$

On the space of functions which are zero on the boundary of $\widehat{\Lambda}_{N} \times \widehat{\Lambda}_{N}$, the generator $B^{N}$ is symmetric with respect to $\left\langle\langle\cdot, \cdot\rangle_{N \times N}\right.$, i.e.,

$$
\left\langle\left\langle f, B^{N} g\right\rangle_{N \times N}=\left\langle\left\langle B^{N} f, g\right\rangle\right\rangle_{N \times N}\right.
$$

for all $f, g: \widehat{\Lambda}_{N} \times \widehat{\Lambda}_{N} \rightarrow \mathbb{R}$ such that $f(0, \cdot)=f(N, \cdot)=f(\cdot, 0)=f(\cdot, N) \equiv 0$ and, analogously, for $g$.

2.3. Test function spaces. In this section, we present, depending on the values of the parameter $\beta \geq 0$, the test function spaces needed to uniquely characterize the weak solution of the limiting hydrodynamic equations. The test function spaces we consider are nuclear Fréchet spaces $\mathscr{S}$ and the solutions will take values in their dual space of tempered distributions $\mathscr{S}^{\prime}$. The construction is standard and follows the ideas in, e.g., [35, Chapter 11] and [34, Chapter 1] of constructing a nested family of Hilbert spaces $\mathcal{H}_{k}, k \in \mathbb{Z}$, with $\mathcal{H}_{0}=L^{2}([0,1])$ and for which the canonical embeddings $\mathcal{H}_{k+k_{*}} \hookrightarrow \mathcal{H}_{k}$ are Hilbert-Schmidt for some $k_{*} \in \mathbb{N}$ and for all $k \in \mathbb{Z}$. The main difference in our context compared to the setting in [35. Chapter 11] is that, for different values of $\beta \geq 0$, different self-adjoint extensions of the Laplacian - corresponding to different boundary conditions - must be employed. We present some essential properties of such spaces in Proposition 2.2 below and leave the details of their construction to Appendix A below.

We acknowledge that several choices of test function spaces (and, thus, of weak solutions to the corresponding PDEs, see Section 3 below) have been employed in the hydrodynamic limit literature. For instance, in [29, more standard Sobolev spaces satisfying an energy estimate and boundary conditions are considered. By taking the aforementioned nuclear space $\mathscr{S}$ as space of test functions, we make a different choice. This is mainly motivated by the fact that we aim at a unified setting for both hydrodynamics and fluctuation results (to be considered in a future work). In fact, on the one 
hand, this setting is certainly considered to be the natural one for the study of fluctuations (see e.g. [35. Chapter 11]); on the other hand, hydrodynamic results are available in this same framework in a number of works, see, e.g., [13] and references therein. Moreover, our construction of such spaces is different from the one used in related publications (see e.g. 24, 25, 30, 6]): there, the authors first define a candidate space of test functions and then verify, knowing some explicit information on suitable orthonormal bases of eigenfunctions, their nuclear structure. In our approach, we first build such spaces from abstract self-adjoint Laplacians and then extract properties of the test functions, without the need of fully characterizing this space. We believe this latter approach to be best suited for proving scaling limits on more general geometries.

In what follows, we distinguish between three different regimes depending on the values of the parameter $\beta \geq 0(\beta<1, \beta=1$ and $\beta>1)$ corresponding, respectively, to Dirichlet, Robin and Neumann boundary conditions.

Proposition 2.2. For each of the three regimes, $\beta<1, \beta=1$ and $\beta>1$, there exists a nuclear Fréchet space $\mathscr{S}=\mathscr{S}_{\beta}$ which continuously embeds into $L^{2}([0,1])$ and which consists of $\mathcal{C}^{\infty}([0,1])$ functions, i.e., smooth functions in $(0,1)$ whose derivatives of all orders admit a continuous extension to $[0,1]$. Moreover, depending on the values of $\beta \geq 0$, the test functions in $\mathscr{S}$ satisfy the following boundary conditions:

Dirichlet $(\beta<1)$. If $G \in \mathscr{S}$, then

$$
\left.\left(\frac{\mathrm{d}^{+}}{\mathrm{d} u}\right)^{2 \ell}\right|_{u=0} G=\left.\left(\frac{\mathrm{d}^{-}}{\mathrm{d} u}\right)^{2 \ell}\right|_{u=1} G=0
$$

holds for all $\ell \in \mathbb{N}_{0}$.

Robin $(\beta=1)$. If $G \in \mathscr{S}$, then

$$
\begin{aligned}
& \left.\left(\frac{\mathrm{d}^{+}}{\mathrm{d} u}\right)^{2 \ell+1}\right|_{u=0} G=\left.\frac{\alpha_{L}}{\alpha}\left(\frac{\mathrm{d}^{+}}{\mathrm{d} u}\right)^{2 \ell}\right|_{u=0} G \\
& \left.\left(\frac{\mathrm{d}^{-}}{\mathrm{d} u}\right)^{2 \ell+1}\right|_{u=1} G=\left.\frac{\alpha_{R}}{\alpha}\left(\frac{\mathrm{d}^{-}}{\mathrm{d} u}\right)^{2 \ell}\right|_{u=1} G
\end{aligned}
$$

holds for all $\ell \in \mathbb{N}_{0}$.

Neumann $(\beta>1)$. If $G \in \mathscr{S}$, then

$$
\left.\left(\frac{\mathrm{d}^{+}}{\mathrm{d} u}\right)^{2 \ell+1}\right|_{u=0} G=\left.\left(\frac{\mathrm{d}^{-}}{\mathrm{d} u}\right)^{2 \ell+1}\right|_{u=1} G=0
$$

holds for all $\ell \in \mathbb{N}_{0}$.

We defer the proof of the above proposition to Appendix A

\section{HydrodynAmiC AND HYDROSTATIC LIMITS}

In this section, we make precise the notion of weak solution to the hydrodynamic equations which we use all throughout (Definition 3.1 below); then, we present the statements of both hydrodynamic and hydrostatic limits for the open SIP (Theorems 3.1 and 3.2 , resp., below).

Let us recall that, for all $\beta \geq 0, \mathscr{S}^{\prime}=\mathscr{S}_{\beta}^{\prime}$ denotes the strong topological dual of $\mathscr{S}=\mathscr{S}_{\beta}$, the space of test functions introduced in Section 2.3 above. Since we state our hydrodynamic and hydrostatic limits in terms of convergence in the space of distributions, we characterize these limits 
as the unique solutions in $\mathscr{S}^{\prime}$ of the following formal partial differential equations:

$$
\left\{\begin{aligned}
\partial_{t} \vartheta(t, u) & =\alpha \partial_{u}^{2} \vartheta(t, u) & & t \in[0, \infty), u \in(0,1) \\
\gamma_{L} \partial_{u}^{+} \vartheta(t, 0) & =\lambda_{L} \vartheta(t, 0)+\sigma_{L} & & t \in(0, \infty) \\
\gamma_{R} \partial_{u}^{-} \vartheta(t, 1) & =\lambda_{R} \vartheta(t, 1)+\sigma_{R} & & t \in(0, \infty) \\
\vartheta(0, u) & =\vartheta_{0}(u) & & u \in[0,1],
\end{aligned}\right.
$$

where $\gamma_{L}, \gamma_{R}, \lambda_{L}, \lambda_{R}, \sigma_{L}, \sigma_{R} \in \mathbb{R}$ are determined according to the value of $\beta \geq 0$ and the system parameters. In particular, if $\beta<1$, then we will recover Dirichlet boundary conditions with

$$
\gamma_{L}=\gamma_{R}=0, \quad \lambda_{L}=\lambda_{R}=1, \quad \sigma_{L}=\vartheta_{L}, \quad \sigma_{R}=\vartheta_{R} ;
$$

if $\beta=1$, we will recover Robin boundary conditions with

$$
\gamma_{L}=\gamma_{R}=\alpha, \quad \lambda_{L}=\alpha_{L}, \quad \lambda_{R}=\alpha_{R}, \quad \sigma_{L}=-\alpha_{L} \vartheta_{L}, \quad \sigma_{R}=-\alpha_{R} \vartheta_{R} ;
$$

if $\beta>1$, we will recover Neumann boundary conditions with

$$
\gamma_{L}=\gamma_{R}=1, \quad \lambda_{L}=\lambda_{R}=\sigma_{L}=\sigma_{R}=0
$$

If we let $h$ denote a stationary solution - not necessarily unique - of the boundary Cauchy problem (3.1), then $\vartheta$ in (3.1) above decomposes as $\vartheta=h+g$, where $g$ formally satisfies

$$
\left\{\begin{aligned}
\partial_{t} g(t, u) & =\alpha \partial_{u}^{2} g(t, u) & & t \in[0, \infty), u \in(0,1) \\
\gamma_{L} \partial_{u}^{+} g(t, 0) & =\lambda_{L} g(t, 0) & & t \in(0, \infty) \\
\gamma_{R} \partial_{u}^{-} g(t, 1) & =\lambda_{R} g(t, 1) & & t \in(0, \infty) \\
g(0, u) & =\vartheta_{0}(u)-h(u) & & u \in[0,1] .
\end{aligned}\right.
$$

Before presenting the precise definition of solutions in $\mathscr{S}^{\prime}$, we need to introduce some notation. For all $G \in \mathscr{S}$ and $g \in \mathscr{S}^{\prime}$, we define $\langle G, g\rangle:=g(G)$. We note that $L^{2}([0,1]) \subsetneq \mathscr{S}^{\prime}$ and, if, e.g., $g \in L^{2}([0,1])$, then $\langle G, g\rangle$ is the usual inner product in $L^{2}([0,1])$. Moreover, we let $\mathcal{C}\left([0, \infty), \mathscr{S}^{\prime}\right)$ and $\mathcal{D}\left([0, \infty), \mathscr{S}^{\prime}\right)$ denote the spaces of $\mathscr{S}^{\prime}$-valued continuous and càdlàg, respectively, functions on $[0, \infty)$ (see, e.g., [34, §2.4], as well as, Appendix Abelow). Finally, for all $\beta \geq 0, \mathcal{A}: \mathscr{S} \rightarrow \mathscr{S}$ denotes the bounded linear operator introduced in Appendix A below, which acts on smooth functions $G \in \mathcal{C}^{\infty}([0,1])$ simply as the rescaled Laplacian

$$
\mathcal{A} G=\alpha \partial_{u}^{2} G
$$

Definition 3.1 (Solutions In $\mathscr{S}^{\prime}$ ). Let $\beta \geq 0$. Given $\vartheta_{0} \in \mathscr{S}^{\prime}$, we say that $\{\vartheta(t): t \in[0, \infty)\} \subset$ $\mathscr{S}^{\prime}$ is a solution of the Dirichlet, Robin or Neumann problem - depending on whether $\beta<1, \beta=1$ or $\beta>1$, respectively - with initial condition $\vartheta_{0}$ if there exists $\{g(t): t \in[0, \infty)\} \subset \mathscr{S}^{\prime}$ for which, for all $G \in \mathscr{S}$ and for all times $t \geq 0$, the following two identities hold:

$$
\langle G, \vartheta(t)\rangle=\left\langle G, h_{\vartheta_{L}, \vartheta_{R}} \mathrm{~d} u\right\rangle+\langle G, g(t)\rangle .
$$

and

$$
\langle G, g(t)\rangle=\left\langle G,\left(\vartheta_{0}-h_{\vartheta_{L}, \vartheta_{R}} \mathrm{~d} u\right)\right\rangle+\int_{0}^{t}\langle\mathcal{A} G, g(s)\rangle \mathrm{d} s .
$$

In the above expressions, $h_{\vartheta_{L}, \vartheta_{R}} \mathrm{~d} u \in \mathscr{S}^{\prime}$ is the distribution that is absolutely continuous with respect to Lebesgue and whose density $h_{\vartheta_{L}, \vartheta_{R}} \in \mathcal{C}^{\infty}([0,1])$ is a stationary solution to (3.1), i.e., given by

$$
h_{\vartheta_{L}, \vartheta_{R}}(u)=\vartheta_{L}+\left(\vartheta_{R}-\vartheta_{L}\right) u, \quad u \in[0,1]
$$

if $\beta<1$, by

$$
h_{\vartheta_{L}, \vartheta_{R}}(u)=\vartheta_{L}+\left(\vartheta_{R}-\vartheta_{L}\right)\left(\frac{\frac{\alpha}{\alpha_{L}}+u}{\frac{\alpha}{\alpha_{L}}+1+\frac{\alpha}{\alpha_{R}}}\right), \quad u \in[0,1]
$$


if $\beta=1$, and by

$$
h_{\vartheta_{L}, \vartheta_{R}}(u)=\vartheta_{L}+\left(\vartheta_{R}-\vartheta_{L}\right) \frac{\alpha_{R}}{\alpha_{L}+\alpha_{R}}=\frac{\rho_{L}+\rho_{R}}{\alpha_{L}+\alpha_{R}}, \quad u \in[0,1],
$$

if $\beta>1$.

As a consequence of the construction of the test function spaces in Section 2.3 (see also Appen$\operatorname{dix}$ A and the theory of generalized Ornstein-Uhlenbeck processes (see, e.g., [31]) applied to this deterministic setting, the following existence and uniqueness result holds.

Proposition 3.1 (Well-Posedness of Hydrodynamic EQUATIOns in $\mathscr{S}^{\prime}$ ). For all $\beta \geq 0$, the solution in $\mathscr{S}^{\prime}$ with initial condition $\vartheta_{0} \in \mathscr{S}^{\prime}$ as defined in Definition 3.1 exists and is unique in $\mathcal{C}\left([0, \infty), \mathscr{S}^{\prime}\right)$.

Proof. By the construction of the nuclear Fréchet space $\mathscr{S}$ and the consequent properties (I) (IV) in Appendix A, Theorem 1.23 in 31] without noise, i.e., taking $B \equiv 0$, applies.

All throughout, since we state our results in terms of solutions in $\mathscr{S}^{\prime}$ and the investigation of their regularity is not the prominent goal of our work, we refer the interested reader to, e.g., [19] for further details, for instance, on the assumptions on the initial condition which guarantee such solutions to be actually strong ones for (3.1). Further notice that the functional framework that we employ allow us to prove our limit theorems (see Theorems 3.1 and 3.2 below) for initial profiles which are generalized functions in $\mathscr{S}^{\prime}$.

3.1. Main results. In this section, we present, for all $\beta \geq 0$, the two results concerning the weak law of large numbers - the hydrodynamic and hydrostatic limits - for the empirical density fields $\left\{\mathscr{X}^{N}: N \in \mathbb{N}\right\} \subset \mathcal{D}\left([0, \infty), \mathscr{S}^{\prime}\right)$, given by

$$
\mathscr{X}_{t}^{N}:=\frac{1}{N} \sum_{x \in \Lambda_{N}} \delta_{\frac{x}{N}} \eta_{t}^{N}(x)=\frac{1}{N} \sum_{x \in \Lambda_{N}} \delta_{\frac{x}{N}} D_{N}\left(x, \eta_{t}^{N}\right) \alpha, \quad t \geq 0,
$$

where, for all $N \in \mathbb{N},\left\{\eta_{t}^{N}: t \geq 0\right\}$ is the open SIP with some prescribed initial distribution $\mu^{N}$ (we refer to the statements of the two main theorems below for further details).

Before the statement of the hydrodynamics result, we need a further definition.

Definition 3.2 (particle distributions associated with a profile). For all $\beta \geq 0$, let $\left\{\mu^{N}: N \in \mathbb{N}\right\}$ be a sequence of Borel probability measures on $\left\{\mathcal{X}_{N}: N \in \mathbb{N}\right\}$ and let $\vartheta_{0} \alpha \in \mathscr{S}^{\prime}$. We say that the family $\left\{\mu^{N}: N \in \mathbb{N}\right\}$ is associated with the profile $\vartheta_{0} \alpha \in \mathscr{S}^{\prime}$ if, for all $G \in \mathscr{S}$ and for all $\delta>0$,

$$
\mu^{N}\left(\left\{\eta \in \mathcal{X}_{N}:\left|\frac{1}{N} \sum_{x \in \Lambda_{N}} G\left(\frac{x}{N}\right) D_{N}(x, \eta) \alpha-\left\langle G, \vartheta_{0} \alpha\right\rangle\right|>\delta\right\}\right) \underset{N \rightarrow \infty}{\longrightarrow} 0 .
$$

Theorem 3.1 (hydrodynamic Limit). For all $\beta \geq 0$, let $\left\{\mu^{N}: N \in \mathbb{N}\right\}$ be a family of Borel probability measures on $\left\{\mathcal{X}_{N}: N \in \mathbb{N}\right\}$ and let $\vartheta_{0} \alpha \in \mathscr{S}^{\prime}$. We assume that:

(a) The family $\left\{\mu^{N}: N \in \mathbb{N}\right\}$ is associated with the profile $\vartheta_{0} \alpha \in \mathscr{S}^{\prime}$ (see Definition 3.2).

(b) There exists a constant $\kappa>0$ such that, for all $N \in \mathbb{N}$ and $x, y \in \widehat{\Lambda}_{N}$, the following upper bounds hold:

$$
E_{\mu^{N}}\left[D_{N}(x, \eta)\right] \leq \kappa \quad \text { and } \quad E_{\mu^{N}}\left[D_{N}(x, y, \eta)\right] \leq \kappa^{2} .
$$

Let us consider the empirical density fields $\left\{\mathscr{X}^{N}: N \in \mathbb{N}\right\} \subset \mathcal{D}\left([0, \infty), \mathscr{S}^{\prime}\right)$ defined as in (3.11) in terms of the open symmetric inclusion processes initialized according to $\left\{\mu^{N}: N \in \mathbb{N}\right\}$, i.e.,

$$
\eta_{0}^{N} \sim \mu^{N}, \quad N \in \mathbb{N} .
$$

Then, the following weak convergence in $\mathcal{D}\left([0, \infty), \mathscr{S}^{\prime}\right)$ (see also 3.15 below)

$$
\left\{\mathscr{X}_{t}^{N}: t \geq 0\right\} \underset{N \rightarrow \infty}{\Longrightarrow}\{\vartheta(t) \alpha: t \geq 0\}
$$


holds, where $\{\vartheta(t) \alpha: t \geq 0\} \in \mathcal{C}\left([0, \infty), \mathscr{S}^{\prime}\right)$ is the unique solution in $\mathscr{S}^{\prime}$ of

- the Dirichlet problem if $\beta<1$,

- the Robin problem if $\beta=1$,

- the Neumann problem if $\beta>1$,

starting from $\vartheta_{0} \alpha \in \mathscr{S}^{\prime}$.

We observe that, if $\vartheta_{0} \alpha \in \mathscr{S}^{\prime}$ is absolutely continuous with respect to Lebesgue with non-negative continuous density $\theta_{0} \alpha \in \mathcal{C}([0,1])$, then the local Gibbs measures $\left\{\mu^{N}: N \in \mathbb{N}\right\}$ (see Section 2.1.1) given by

$$
\mu^{N}=\otimes_{x \in \Lambda_{N}} \nu_{\theta_{0}\left(\frac{x}{N}\right)}, \quad N \in \mathbb{N},
$$

satisfy both assumptions (a) with the profile $\vartheta_{0} \alpha$ and $(\mathrm{b})$ with $\kappa:=\max \left\{\vartheta_{L}, \vartheta_{R}, \sup _{u \in[0,1]} \theta_{0}(u)\right\}$.

Furthermore, because the transitions of the underlying open particle system consist in only oneparticle moves, for all $\beta \geq 0, G \in \mathscr{S}$ and $\delta>0$, we have

$$
\mathbb{P}_{\mu^{N}}^{N}\left(\sup _{t \geq 0}\left|\left\langle G, \mathscr{X}_{t}^{N}\right\rangle-\left\langle G, \mathscr{X}_{t^{-}}^{N}\right\rangle\right|>\delta\right) \underset{N \rightarrow \infty}{\longrightarrow} 0
$$

from which it follows $\left(\left[\underline{8}\right.\right.$, Theorem 13.4]) that any limiting point $\mathscr{X}$. of the sequence $\left\{\mathscr{X}^{N}: N \in \mathbb{N}\right\}$ belongs to $\mathcal{C}\left([0, \infty), \mathscr{S}^{\prime}\right)$. The result in Proposition 3.1 and assumption (a) will, then, univocally characterize the deterministic limiting process.

The weak convergence in (3.13), which boils down to show tightness and convergence of the finite dimensional distributions of the sequence $\left\{\mathscr{X}^{N}: N \in \mathbb{N}\right\}$ (see, e.g., [41, Proposition 5.2]), because of the considerations in Section A.1 below and because the limiting process is deterministic and continuous, may be equivalently restated as follows (cf. A.12 below, as well as, e.g., 34. for further details): for all $T \geq 0, G \in \mathscr{S}$ and $\delta>0$,

$$
\mathbb{P}_{\mu^{N}}^{N}\left(\sup _{t \in[0, T]}\left|\left\langle G, \mathscr{X}_{t}^{N}\right\rangle-\langle G, \vartheta(t) \alpha\rangle\right|>\delta\right) \underset{N \rightarrow \infty}{\longrightarrow} 0 .
$$

The general strategy we follow to prove Theorem 3.1 is to, first, provide a decomposition for the empirical density fields analogous to that in 3.6 in which we center the fields with respect to the stationary part, i.e., write, for all $N \in \mathbb{N}$ and $t \geq 0$,

$$
\mathscr{X}_{t}^{N}=\mathscr{H}_{\vartheta_{L}, \vartheta_{R}}^{N} \alpha+\mathscr{Z}_{t}^{N}
$$

for $\mathscr{H}_{\vartheta_{L} \vartheta_{R}}^{N} \in \mathscr{S}^{\prime}$ deterministic and $\left\{\mathscr{Z}_{t}^{N}: t \geq 0\right\} \in \mathcal{D}\left([0, \infty), \mathscr{S}^{\prime}\right)$ random; then, show that

$$
\left\langle G, \mathscr{H}_{\vartheta_{L}, \vartheta_{R}}^{N} \alpha\right\rangle \underset{N \rightarrow \infty}{\longrightarrow}\left\langle G, h_{\vartheta_{L}, \vartheta_{R}} \alpha \mathrm{d} u\right\rangle
$$

and

$$
\mathbb{P}_{\mu^{N}}^{N}\left(\sup _{t \in[0, T]}\left|\left\langle G, \mathscr{Z}_{t}^{N}\right\rangle-\left\langle G,\left(\vartheta(t)-h_{\vartheta_{L}, \vartheta_{R}} \mathrm{~d} u\right) \alpha\right\rangle\right|>\delta\right) \underset{N \rightarrow \infty}{\longrightarrow} 0
$$

hold for all $T \geq 0, G \in \mathscr{S}$ and $\delta>0$.

In the following theorem, we present our second main result and recall that, for all $\beta \geq 0$ and $N \in \mathbb{N}, \mu_{\vartheta_{L}, \vartheta_{R}}^{N}$ denotes the unique stationary probability measure for the open SIP $\left\{\eta_{t}^{N}: t \geq 0\right\}$ (see Section 2.1.1).

Theorem 3.2 (hydrostatic LIMIT). For all $\beta \geq 0$, the empirical density fields - given in (3.11) and defined in terms of the open $\mathrm{SIP}$ initialized according to $\left\{\mu_{\vartheta_{L}, \vartheta_{R}}^{N}: N \in \mathbb{N}\right\}-$ weakly converge in $\mathcal{D}\left([0, \infty), \mathscr{S}^{\prime}\right)$ to $\{\vartheta(t) \alpha: t \geq 0\} \in \mathcal{C}\left([0, \infty), \mathscr{S}^{\prime}\right)$, where $\vartheta(t) \equiv h_{\vartheta_{L}, \vartheta_{R}} \mathrm{~d} u \in \mathscr{S}^{\prime}$ and $h_{\vartheta_{L}, \vartheta_{R}} \in$ $\mathcal{C}^{\infty}([0,1])$ is the unique stationary solution of

- the Dirichlet problem as given in 3.8 if $\beta<1$, 
- the Robin problem as given in 3.9 if $\beta=1$,

- the Neumann problem as given in 3.10 if $\beta>1$.

\section{Proofs}

Let us now prove Theorems 3.1 and 3.2. Before digging into the proofs, though, we start with some general considerations.

Because of duality (2.8) and because the dual process defined in Section 2.2.1 conserves the total number of particles, in view of assumption (b) in Theorem 3.1 we have

$$
\sup _{N \in \mathbb{N}} \sup _{t \geq 0} \sup _{x \in \widehat{\Lambda}_{N}} \mathbb{E}_{\mu^{N}}^{N}\left[D_{N}\left(x, \eta_{t}^{N}\right)\right] \leq \kappa \quad \text { and } \sup _{N \in \mathbb{N}} \sup _{t \geq 0} \sup _{x, y \in \widehat{\Lambda}_{N}} \mathbb{E}_{\mu^{N}}^{N}\left[D_{N}\left(x, y, \eta_{t}^{N}\right)\right] \leq \kappa^{2} .
$$

We recall the definition of the empirical density fields in (3.11). In view of Dynkin's formula, for all $\beta \geq 0, N \in \mathbb{N}, t \geq 0$ and $G \in \mathscr{S}$, we have

$$
\left\langle G, \mathscr{X}_{t}^{N}\right\rangle=\left\langle G, \mathscr{X}_{0}^{N}\right\rangle+\int_{0}^{t} \mathcal{L}^{N}\left\langle G, \mathscr{X}_{s}^{N}\right\rangle \mathrm{d} s+\left\langle G, \mathscr{M}_{t}^{N}\right\rangle
$$

where $\left\{\mathscr{M}_{t}^{N}: t \geq 0\right\} \subset \mathcal{D}\left([0, \infty), \mathscr{S}^{\prime}\right)$ is a martingale (with respect to its natural filtration) with predictable quadratic variation given, for all $t \geq 0$, by

$$
\int_{0}^{t}\left(\mathcal{L}^{N}\left(\left\langle G, \mathscr{X}_{s}^{N}\right\rangle\right)^{2}-2\left\langle G, \mathscr{X}_{s}^{N}\right\rangle \mathcal{L}^{N}\left\langle G, \mathscr{X}_{s}^{N}\right\rangle\right) \mathrm{d} s
$$

We recall the definition of the inner product $\langle\cdot \cdot \cdot \cdot\rangle_{N}$ from 2.12 and of the generator $A^{N}$ in 2.10). In view of the duality relations 2.8 and, in particular, 2.9) and 2.13), we have

$$
\mathcal{L}^{N}\left\langle G, \mathscr{X}_{s}^{N}\right\rangle=\frac{1}{N} \sum_{x \in \Lambda_{N}} G\left(\frac{x}{N}\right) A^{N} D_{N}\left(\cdot, \eta_{s}^{N}\right)(x) \alpha=\left\langle\left\langle G(\dot{\bar{N}}), A^{N} D_{N}\left(\cdot, \eta_{s}^{N}\right)\right\rangle_{N}\right.
$$

and

$$
\begin{aligned}
\int_{0}^{t}\left(\mathcal{L}^{N}\left(\left\langle G, \mathscr{X}_{s}^{N}\right\rangle\right)^{2}-2\left\langle G, \mathscr{X}_{s}^{N}\right\rangle \mathcal{L}^{N}\left\langle G, \mathscr{X}_{s}^{N}\right\rangle\right) \mathrm{d} s \\
=\frac{1}{N^{2}} \int_{0}^{t}\left\{\begin{array}{c}
\sum_{x \in \Lambda_{N} \backslash\{N-1\}} N^{2} \alpha\left(G\left(\frac{x+1}{N}\right)-G\left(\frac{x}{N}\right)\right)^{2} \mathcal{V}_{\{x, x+1\}}^{N}\left(\eta_{s}^{N}\right) \alpha \\
+N^{2-\beta} \alpha_{L}\left(G\left(\frac{1}{N}\right)\right)^{2} \mathcal{V}_{\{0,1\}}^{N}\left(\eta_{s}^{N}\right) \alpha \\
+N^{2-\beta} \alpha_{R}\left(G\left(\frac{N-1}{N}\right)\right)^{2} \mathcal{V}_{\{N-1, N\}}^{N}\left(\eta_{s}^{N}\right) \alpha
\end{array}\right\} \mathrm{d} s,
\end{aligned}
$$

where, for all $\eta \in \mathcal{X}_{N}$ and $x, y \in \widehat{\Lambda}_{N}$ with $x \neq y$,

$$
\mathcal{V}_{\{x, y\}}^{N}(\eta):=D_{N}(x, \eta)+D_{N}(y, \eta)+2 D_{N}(x, y, \eta) .
$$

Going back to 4.3 , we note that, for all $f: \widehat{\Lambda}_{N} \rightarrow \mathbb{R}$, two applications of integration by parts yield

$$
\begin{aligned}
\left\langle\left\langle G\left(\frac{\dot{\bar{N}}}{N}\right), A^{N} f\right\rangle_{N}\right. & =\frac{1}{N} \sum_{x \in \Lambda_{N}} \alpha \Delta_{N} G\left(\frac{x}{N}\right) f(x) \alpha \\
& +\alpha \nabla_{N}^{+} G(0) f(1) \alpha+\alpha \nabla_{N}^{-} G(1) f(N-1) \alpha \\
& +N^{1-\beta} \alpha_{L} G\left(\frac{1}{N}\right)(f(0)-f(1)) \alpha+N^{1-\beta} \alpha_{R} G\left(\frac{N-1}{N}\right)(f(N)-f(N-1)) \alpha,
\end{aligned}
$$

where $\Delta_{N}$ denotes the discrete Laplacian with mesh size $\frac{1}{N}$, namely

$$
\Delta_{N} G\left(\frac{x}{N}\right):=N^{2}\left(G\left(\frac{x+1}{N}\right)+G\left(\frac{x-1}{N}\right)-2 G\left(\frac{x}{N}\right)\right), \quad x \in \Lambda_{N},
$$


and $\nabla_{N}^{ \pm}$the corresponding discrete gradients:

$$
\begin{array}{ll}
\nabla_{N}^{+} G\left(\frac{x}{N}\right):=N\left(G\left(\frac{x+1}{N}\right)-G\left(\frac{x}{N}\right)\right), & x \in \widehat{\Lambda}_{N} \backslash\{N\} \\
\nabla_{N}^{-} G\left(\frac{x}{N}\right):=N\left(G\left(\frac{x-1}{N}\right)-G\left(\frac{x}{N}\right)\right), & x \in \widehat{\Lambda}_{N} \backslash\{0\} .
\end{array}
$$

We recall from 2.11 that if, additionally, $G(0)=G(1)=0$ and $f(0)=f(N)=0$, then

$$
\left\langle\left\langle G(\dot{\bar{N}}), A^{N} f\right\rangle\right\rangle_{N}=\left\langle\left\langle\mathcal{A}^{N} G(\dot{\bar{N}}), f\right\rangle_{N},\right.
$$

where

$$
\mathcal{A}^{N} G\left(\frac{x}{N}\right):=A^{N} G(\dot{\bar{N}})(x), \quad x \in \widehat{\Lambda}_{N} .
$$

Having in mind the decomposition in (3.16) of the empirical density fields, we introduce, for all $\beta \geq 0$ and $N \in \mathbb{N}$, the following function

$$
h_{\vartheta_{L}, \vartheta_{R}}^{N}(x):=E_{\mu_{\vartheta_{L}, \vartheta_{R}}^{N}}\left[D_{N}(x, \eta)\right]=\lim _{t \rightarrow \infty} \mathbb{E}_{\mu^{N}}\left[D_{N}\left(x, \eta_{t}^{N}\right)\right], \quad x \in \widehat{\Lambda}_{N}
$$

for any probability measure $\mu^{N}$ on $\mathcal{X}_{N}$, which, by stationarity of $\mu_{\vartheta_{L}, \vartheta_{R}}^{N}$ and duality [2.9), solves the following boundary value problem:

$$
\left\{\begin{aligned}
A^{N} f(x) & =0, \quad x \in \widehat{\Lambda}_{N} \\
f(0) & =\vartheta_{L} \\
f(N) & =\vartheta_{R} .
\end{aligned}\right.
$$

Notice that, as we will show in Lemma 4.1 below, the functions $h_{\vartheta_{L}, \vartheta_{R}}^{N}$ are to be considered as discrete approximations of the stationary solutions of the hydrodynamic equations. Moreover, by defining

$$
\mathscr{H}_{\vartheta_{L}, \vartheta_{R}}^{N} \alpha:=\frac{1}{N} \sum_{x \in \Lambda_{N}} \delta_{\frac{x}{N}} h_{\vartheta_{L}, \vartheta_{R}}^{N}(x) \alpha
$$

(3.16) writes, for all $\beta \geq 0, N \in \mathbb{N}, t \geq 0$ and $G \in \mathscr{S}$, as

$$
\begin{aligned}
\left\langle G, \mathscr{X}_{t}^{N}\right\rangle & =\left\langle G, \mathscr{H}_{\vartheta_{L}, \vartheta_{R}}^{N} \alpha\right\rangle+\left\langle G, \mathscr{Z}_{t}^{N}\right\rangle \\
& =\left\langle\left\langle G(\dot{\bar{N}}), h_{\vartheta_{L}, \vartheta_{R}}^{N}\right\rangle_{N}+\frac{1}{N} \sum_{x \in \Lambda_{N}} G\left(\frac{x}{N}\right)\left(\frac{\eta_{t}^{N}(x)}{\alpha}-h_{\vartheta_{L}, \vartheta_{R}}^{N}(x)\right) \alpha\right. \\
& =\left\langle\left\langle G(\dot{\bar{N}}), h_{\vartheta_{L}, \vartheta_{R}}^{N}\right\rangle_{N}+\frac{1}{N} \sum_{x \in \Lambda_{N}} G\left(\frac{x}{N}\right)\left(D_{N}\left(x, \eta_{t}^{N}\right)-E_{\mu_{\vartheta_{L}, \vartheta_{R}}^{N}}\left[D_{N}(x, \eta)\right]\right) \alpha .\right.
\end{aligned}
$$

In our one-dimensional context, the explicit form of the function $h_{\vartheta_{L}, \vartheta_{R}}^{N}$ is well-known and given by

$$
h_{\vartheta_{L}, \vartheta_{R}}^{N}(x)=\vartheta_{L}+p_{N}(x)\left(\vartheta_{R}-\vartheta_{L}\right), \quad x \in \widehat{\Lambda}_{N},
$$

with

$$
p_{N}(x):=\frac{\mathbf{1}_{\{x>0\}}}{Z^{N}} \frac{1}{N}\left(\frac{1}{N^{-\beta} \alpha_{L} \alpha}+\frac{x-1}{\alpha^{2}}+\mathbf{1}_{\{x=N\}} \frac{1}{N^{-\beta} \alpha_{R} \alpha}\right)
$$

where

$$
Z^{N}:=\frac{1}{N}\left(\frac{1}{N^{-\beta} \alpha_{L} \alpha}+\frac{N-1}{\alpha^{2}}+\frac{1}{N^{-\beta} \alpha_{R} \alpha}\right) .
$$

In particular, $h_{\vartheta_{L}, \vartheta_{R}}^{N}(0)=\vartheta_{L}$ and $h_{\vartheta_{L}, \vartheta_{R}}^{N}(N)=\vartheta_{R}$. Hence, in order to prove both Theorems 3.1 and 3.2 as a first step, we prove the convergence in (3.17) in the following lemma. 
Lemma 4.1 (CONVERGENCE OF THE STATIONARY PART). Let us recall, for all $\beta \geq 0$, the definitions of stationary solutions $h_{\vartheta_{L}, \vartheta_{R}}$ in 23.8), 3.9) and 3.10) as well as the definition of $\mathscr{H}_{\vartheta_{L}, \vartheta_{R}}^{N}$ in (4.10). Then, for all $\beta \geq 0$ and $G \in \mathscr{S}$, we have

$$
\left\langle G, \mathscr{H}_{\vartheta_{L}, \vartheta_{R}}^{N} \alpha\right\rangle \underset{N \rightarrow \infty}{\longrightarrow}\left\langle G, h_{\vartheta_{L}, \vartheta_{R}} \alpha \mathrm{d} u\right\rangle=\int_{[0,1]} G(u) h_{\vartheta_{L}, \vartheta_{R}}(u) \alpha \mathrm{d} u .
$$

Proof. We note that, for all $\beta \geq 0$ and by definition of $h_{\vartheta_{L}, \vartheta_{R}}^{N}$ in 4.12 , we have

$$
\sup _{x \in \Lambda_{N}}\left|h_{\vartheta_{L}, \vartheta_{R}}\left(\frac{x}{N}\right)-h_{\vartheta_{L}, \vartheta_{R}}^{N}(x)\right| \underset{N \rightarrow \infty}{\longrightarrow} 0 \text {. }
$$

Combined with the integrability of $G \in \mathscr{S}$, this concludes the proof.

In what follows, for each of the three regimes $\beta<1, \beta=1$ and $\beta>1$, we conclude the proof of Theorem 3.1 by proving (3.18) for the processes $\left\{\mathscr{Z}^{N}: N \in \mathbb{N}\right\} \subset \mathcal{D}\left([0, \infty), \mathscr{S}^{\prime}\right)$ given in (4.11), for all $N \in \mathbb{N}$ and $t \geq 0$, as

$$
\mathscr{Z}_{t}^{N}=\frac{1}{N} \sum_{x \in \Lambda_{N}} \delta_{\frac{x}{N}}\left(\frac{\eta_{t}^{N}(x)}{\alpha}-h_{\vartheta_{L}, \vartheta_{R}}^{N}(x)\right) \alpha .
$$

Moreover, because $h_{\vartheta_{L}, \vartheta_{R}}^{N}$ is harmonic for $A^{N}$, we have

$$
\mathcal{L}^{N} h_{\vartheta_{L}, \vartheta_{R}}^{N}(x)=A^{N} h_{\vartheta_{L}, \vartheta_{R}}^{N}(x)=0, \quad x \in \widehat{\Lambda}_{N},
$$

and, hence,

$$
\mathcal{L}^{N}\left\langle G, \mathscr{Z}_{t}^{N}\right\rangle=\frac{1}{N} \sum_{x \in \Lambda_{N}} G\left(\frac{x}{N}\right) A^{N}\left(D_{N}\left(\cdot, \eta_{t}^{N}\right)-h_{\vartheta_{L}, \vartheta_{R}}^{N}(\cdot)\right)(x) \alpha
$$

We further remark that, because $\left\{\mathscr{X}_{t}^{N}: t \geq 0\right\}$ and $\left\{\mathscr{Z}_{t}^{N}: t \geq 0\right\}$ differ only by a deterministic term, the corresponding martingales arising from Dynkin's decomposition coincide.

In conclusion, from the following identity

$$
\begin{aligned}
& \left(D_{N}(x, \eta)-h_{\vartheta_{L}, \vartheta_{R}}^{N}(x)\right)\left(D_{N}(y, \eta)-h_{\vartheta_{L}, \vartheta_{R}}^{N}(y)\right) \alpha^{2} \\
= & D_{N}(x, y, \eta) \alpha\left(\alpha+\mathbf{1}_{\{x=y\}}\right)-D_{N}(x, \eta) h_{\vartheta_{L}, \vartheta_{R}}^{N}(y) \alpha^{2}-D_{N}(y, \eta) h_{\vartheta_{L}, \vartheta_{R}}^{N}(x) \alpha^{2} \\
+ & h_{\vartheta_{L}, \vartheta_{R}}^{N}(x) h_{\vartheta_{L}, \vartheta_{R}}^{N}(y) \alpha^{2}+\mathbf{1}_{\{x=y\}} D_{N}(x, \eta) \alpha, \quad x, y \in \Lambda_{N}
\end{aligned}
$$

we obtain, for all $\beta \geq 0, N \in \mathbb{N}, G \in \mathscr{S}$ and $t \geq 0$,

$$
\begin{aligned}
& \left(\left\langle G, \mathscr{Z}_{t}^{N}\right\rangle\right)^{2} \\
= & \frac{1}{N^{2}} \sum_{x \in \Lambda_{N}} \sum_{y \in \Lambda_{N}} G\left(\frac{x}{N}\right) G\left(\frac{y}{N}\right)\left(D_{N}\left(x, \eta_{t}^{N}\right)-h_{\vartheta_{L}, \vartheta_{R}}^{N}(x)\right)\left(D_{N}\left(y, \eta_{t}^{N}\right)-h_{\vartheta_{L}, \vartheta_{R}}^{N}(y)\right) \alpha^{2} \\
= & \left.\left\langle(G \otimes G)\left(\frac{\dot{\bar{N}}}{\dot{\bar{N}}}\right), D_{N}\left(\cdot, \cdot, \eta_{t}^{N}\right)\right\rangle\right\rangle_{N \times N}-2\left\langle\langle G ( \dot { \dot { N } } ) , D _ { N } ( \cdot , \eta _ { t } ^ { N } ) \rangle _ { N } \left\langle\left\langle G(\dot{\bar{N}}), h_{\vartheta_{L}, \vartheta_{R}}^{N}\right\rangle_{N}\right.\right. \\
+ & \left(\left\langle\left\langle G(\dot{\bar{N}}), h_{\vartheta_{L}, \vartheta_{R}}^{N}\right\rangle_{N}\right)^{2}+\frac{1}{N}\left\langle\left\langle G^{2}(\dot{\bar{N}}), D_{N}\left(\cdot, \eta_{t}^{N}\right)\right\rangle_{N} .\right.\right.
\end{aligned}
$$

\subsection{Proof of Theorem 3.1.}

4.1.1. Case $\beta \geq 1$. Let us recall the definition in (4.13). In order to prove Theorem 3.1, we show that the limiting distribution of the fields $\left\{\mathscr{Z}^{N}: N \in \mathbb{N}\right\}$ is fully supported on solutions of some integral equations; the uniqueness result in Proposition 3.1 concludes then the proof. More specifically, we prove that, for all $\beta \geq 1, \delta>0, T>0$ and $G \in \mathscr{S}$, we have

$$
\mathbb{P}_{\mu^{N}}^{N}\left(\sup _{t \in[0, T]}\left|\left\langle G, \mathscr{Z}_{t}^{N}\right\rangle-\int_{0}^{t}\left\langle\mathcal{A} G, \mathscr{Z}_{s}^{N}\right\rangle \mathrm{d} s-\left\langle G,\left(\vartheta_{0}-h_{\vartheta_{L}, \vartheta_{R}} \mathrm{~d} u\right) \alpha\right\rangle\right|>\delta\right) \underset{N \rightarrow \infty}{\longrightarrow} 0 .
$$


By Dynkin's formula in 4.2 , the above is equivalent to

$$
\mathbb{P}_{\mu^{N}}^{N}\left(\sup _{t \in[0, T]}\left|\begin{array}{c}
{\left[\left\langle G, \mathscr{Z}_{0}^{N}\right\rangle-\left\langle G,\left(\vartheta_{0}-h_{\vartheta_{L}, \vartheta_{R}} \mathrm{~d} u\right) \alpha\right\rangle\right]} \\
+\left[\int_{0}^{t}\left(\mathcal{L}^{N}\left\langle G, \mathscr{Z}_{s}^{N}\right\rangle-\left\langle\mathcal{A} G, \mathscr{Z}_{s}^{N}\right\rangle\right) \mathrm{d} s\right] \\
+\left[\left\langle G, \mathscr{M}_{t}^{N}\right\rangle\right]
\end{array}\right|>\delta\right) \underset{N \rightarrow \infty}{\longrightarrow} 0 .
$$

Let us prove that each of the three terms in square brackets vanishes uniformly in $[0, T]$ in probability; to this purpose, we follow some of the arguments in [4, Proposition 4.1]. Regarding the first term containing information only about the initial conditions of the fields and the limiting solution, by assumption (a) in Theorem 3.1 and Lemma 4.1, for all $\beta \geq 1$, we have

$$
\mu^{N}\left(\left\{\eta \in \mathcal{X}_{N}:\left|\begin{array}{c}
\frac{1}{N} \sum_{x \in \Lambda_{N}} G\left(\frac{x}{N}\right)\left(\frac{\eta(x)}{\alpha}-h_{\vartheta_{L}, \vartheta_{R}}^{N}(x)\right) \alpha \\
-\int_{[0,1]} G(u)\left(\vartheta_{0}(u)-h_{\vartheta_{L}, \vartheta_{R}}(u)\right) \alpha \mathrm{d} u
\end{array}\right|>\delta\right\}\right) \underset{N \rightarrow \infty}{\longrightarrow} 0
$$

Turning to the second term consisting of a time integral, it suffices to show, by Chebyshev's and Cauchy-Schwarz inequalities, that

$$
\sup _{t \in[0, T]} \mathbb{E}_{\mu^{N}}^{N}\left[\left(\mathcal{L}^{N}\left\langle G, \mathscr{Z}_{t}^{N}\right\rangle-\left\langle\mathcal{A} G, \mathscr{Z}_{t}^{N}\right\rangle\right)^{2}\right] \underset{N \rightarrow \infty}{\longrightarrow} 0
$$

By (4.14) and 4.5), we have

$$
\begin{aligned}
& \mathbb{E}_{\mu^{N}}^{N}\left[\left(\mathcal{L}^{N}\left\langle G, \mathscr{Z}_{t}^{N}\right\rangle-\left\langle\mathcal{A} G, \mathscr{Z}_{t}^{N}\right\rangle\right)^{2}\right] \\
\leq & 2 \mathbb{E}_{\mu^{N}}^{N}\left[\left(\left\langle\alpha \Delta_{N} G-\mathcal{A} G, \mathscr{Z}_{t}^{N}\right\rangle\right)^{2}\right] \\
+ & 4 \mathbb{E}_{\mu^{N}}^{N}\left[\left(\left(\alpha \nabla_{N}^{+} G(0)-N^{1-\beta} \alpha_{L} G\left(\frac{1}{N}\right)\right)\left(D_{N}\left(1, \eta_{t}^{N}\right)-h_{\vartheta_{L}, \vartheta_{R}}^{N}(1)\right) \alpha\right)^{2}\right] \\
+ & 4 \mathbb{E}_{\mu^{N}}^{N}\left[\left(\left(\alpha \nabla_{N}^{-} G(1)-N^{1-\beta} \alpha_{R} G\left(\frac{N-1}{N}\right)\right)\left(D_{N}\left(N-1, \eta_{t}^{N}\right)-h_{\vartheta_{L}, \vartheta_{R}}^{N}(N-1)\right) \alpha\right)^{2}\right] .
\end{aligned}
$$

The first term on the r.h.s. above, by the smoothness of $G \in \mathscr{S}$, the identity 4.16 and the upper bound 4.1, vanishes as $N \rightarrow \infty$. Both the second and third terms on the r.h.s. in 4.20 can be treated analogously; we therefore only give details for the second term. By 4.1] and the identity 4.15) there exists a constant $C=C\left(\kappa, \vartheta_{L}, \vartheta_{R}\right)>0$ for which we have

$$
\begin{aligned}
& \mathbb{E}_{\mu^{N}}^{N}\left[\left(\left(\alpha \nabla_{N}^{+} G(0)-N^{1-\beta} \alpha_{L} G\left(\frac{1}{N}\right)\right)\left(D_{N}\left(1, \eta_{t}^{N}\right)-h_{\vartheta_{L}, \vartheta_{R}}^{N}(1)\right) \alpha\right)^{2}\right] \\
\leq & C \alpha^{2}\left(\alpha \nabla_{N}^{+} G(0)-N^{1-\beta} \alpha_{L} G\left(\frac{1}{N}\right)\right)^{2} .
\end{aligned}
$$

For the case $\beta=1$, because $G \in \mathscr{S}$ is smooth and satisfies the boundary conditions in (2.18), we have

$$
\begin{aligned}
\left(\alpha \nabla_{N}^{+} G(0)-\alpha_{L} G\left(\frac{1}{N}\right)\right)^{2} & \leq 2\left(\alpha \partial_{u}^{+} G(0)-\alpha_{L} G(0)\right)^{2}+\frac{2}{N^{2}}\left(\alpha\left\|\partial_{u}^{2} G\right\|_{\infty}+\alpha_{L}\left\|\partial_{u}^{2} G\right\|_{\infty}\right)^{2} \\
& =\frac{2}{N^{2}}\left(\alpha\left\|\partial_{u}^{2} G\right\|_{\infty}+\alpha_{L}\left\|\partial_{u}^{2} G\right\|_{\infty}\right)^{2} \underset{N \rightarrow \infty}{\longrightarrow} 0
\end{aligned}
$$

with $\|\cdot\|_{\infty}$ denoting the supremum norm on $[0,1]$. For the case $\beta>1, G \in \mathscr{S}$ satisfies the boundary conditions in 2.19), yielding

$$
\begin{aligned}
\left(\alpha \nabla_{N}^{+} G(0)-N^{1-\beta} \alpha_{L} G\left(\frac{1}{N}\right)\right)^{2} & \leq 2\left(\alpha \partial_{u}^{+} G(0)+N^{1-\beta} \alpha_{L}\|G\|_{\infty}\right)^{2}+\frac{2}{N^{2}}\left(\alpha\left\|\partial_{u} G\right\|_{\infty}\right)^{2} \\
& =\frac{2}{N^{2(\beta-1)}}\left(\alpha_{L}\|G\|_{\infty}\right)^{2}+\frac{2}{N^{2}}\left(\alpha\left\|\partial_{u} G\right\|_{\infty}\right)^{2} \underset{N \rightarrow \infty}{\longrightarrow} 0 .
\end{aligned}
$$


This proves 4.19) for all $\beta \geq 1$. We conclude the proof of 4.17 by showing that

$$
\mathbb{E}_{\mu^{N}}^{N}\left[\frac{1}{N^{2}} \int_{0}^{T}\left\{\begin{array}{c}
\sum_{x \in \Lambda_{N} \backslash\{N-1\}} N^{2} \alpha\left(G\left(\frac{x+1}{N}\right)-G\left(\frac{x}{N}\right)\right)^{2} \mathcal{V}_{\{x, x+1\}}^{N}\left(\eta_{s}^{N}\right) \alpha \\
+N^{2-\beta} \alpha_{L}\left(G\left(\frac{1}{N}\right)\right)^{2} \mathcal{V}_{\{0,1\}}^{N}\left(\eta_{s}^{N}\right) \alpha \\
+N^{2-\beta} \alpha_{R}\left(G\left(\frac{N-1}{N}\right)\right)^{2} \mathcal{V}_{\{N-1, N\}}^{N}\left(\eta_{s}^{N}\right) \alpha
\end{array}\right\} \mathrm{d} s\right] \underset{N \rightarrow \infty}{\longrightarrow} 0
$$

where the expression inside the expectation is the predictable quadratic variation of the martingale arising from Dynkin's decomposition of the fields $\left\{\mathscr{Z}^{N}: N \in \mathbb{N}\right\}$, see 4.4 for the definition. Indeed, (4.21) follows because, by (4.1), we have

$$
\sup _{N \in \mathbb{N}} \sup _{x \in \widehat{\Lambda}_{N} \backslash\{N\}} \sup _{t \in[0, T]}\left|\mathbb{E}_{\mu^{N}}^{N} \mathcal{V}_{\{x, x+1\}}^{N}\left(\eta_{s}^{N}\right)\right| \leq C
$$

for some constant $C=C\left(\kappa, \vartheta_{L}, \vartheta_{R}\right)>0$ and, by Fubini,

$$
\begin{aligned}
& \mathbb{E}_{\mu^{N}}^{N}\left[\frac{1}{N^{2}} \int_{0}^{T}\left\{\begin{array}{c}
\sum_{\substack{x \in \Lambda_{N} \\
x \neq N-1}} N^{2} \alpha\left(G\left(\frac{x+1}{N}\right)-G\left(\frac{x}{N}\right)\right)^{2} \mathcal{V}_{\{x, x+1\}}^{N}\left(\eta_{s}^{N}\right) \alpha \\
+N^{2-\beta} \alpha_{L}\left(G\left(\frac{1}{N}\right)\right)^{2} \mathcal{V}_{\{0,1\}}^{N}\left(\eta_{s}^{N}\right) \alpha \\
+N^{2-\beta} \alpha_{R}\left(G\left(\frac{N-1}{N}\right)\right)^{2} \mathcal{V}_{\{N-1, N\}}^{N}\left(\eta_{s}^{N}\right) \alpha
\end{array}\right\} \mathrm{d} s\right] \\
& \leq \frac{C T}{N}\left\{\frac{1}{N} \sum_{\substack{x \in \Lambda_{N} \\
x \neq N-1}}\left(\nabla_{N}^{+} G\left(\frac{x}{N}\right)\right)^{2} \alpha^{2}+N^{1-\beta} \alpha_{L}\left(G\left(\frac{1}{N}\right)\right)^{2} \alpha+N^{1-\beta} \alpha_{R}\left(G\left(\frac{N-1}{N}\right)\right)^{2} \alpha\right\},
\end{aligned}
$$

which vanishes as $N \rightarrow \infty$ because $\beta \geq 1$ and $G \in \mathscr{S}$ is smooth.

4.1.2. Case $\beta \in[0,1)$. Here, compared to the case $\beta \geq 1$, we adopt a different strategy since, due to the higher intensity of the reservoir interaction, we cannot directly prove the claim in (4.17) with the supremum over time. Instead, we prove first convergence of finite dimensional distributions and then tightness for the empirical density fields $\left\{\mathscr{Z}^{N}: N \in \mathbb{N}\right\} \subset \mathcal{D}\left([0, \infty), \mathscr{S}^{\prime}\right)$.

Let $\left\{\vartheta(t)-h_{\vartheta_{L}, \vartheta_{R}} \mathrm{~d} u: t \geq 0\right\}$ be the unique Dirichlet solution with initial condition given by $\vartheta_{0}-h_{\vartheta_{L}, \vartheta_{R}} \mathrm{~d} u$. To the purpose of showing convergence of finite dimensional distributions to those of the deterministic process $\left\{\left(\vartheta(t)-h_{\vartheta_{L}, \vartheta_{R}} \mathrm{~d} u\right) \alpha: t \geq 0\right\} \in \mathcal{C}\left([0, \infty), \mathscr{S}^{\prime}\right)$, it suffices to prove that, for all $\beta \geq 0, t \geq 0, G \in \mathscr{S}$ and $\delta>0$,

$$
\mathbb{P}_{\mu^{N}}^{N}\left(\left|\left\langle G, \mathscr{Z}_{t}^{N}\right\rangle-\left\langle G,\left(\vartheta(t)-h_{\vartheta_{L}, \vartheta_{R}} \mathrm{~d} u\right) \alpha\right\rangle\right|>\delta\right) \underset{N \rightarrow \infty}{\longrightarrow} 0
$$

holds true. Notice again that, compared to 4.17), the supremum over time does not appear in the displacement above. Instead of proving (4.22 directly, we introduce an auxiliary process reminiscent of the so-called corrected empirical density field (see, e.g., 32] - whose finite dimensional distributions approximate those of the empirical fields $\left\{\mathscr{Z}^{N}: N \in \mathbb{N}\right\}$ and for which this convergence follows right away. First, we need to prove the following lemma.

Lemma 4.2. For all $\beta \in[0,1)$ and $G \in \mathscr{S}$, there exists a sequence of functions

$$
\left\{G_{N}: N \in \mathbb{N}\right\}, \quad G_{N}: \widehat{\Lambda}_{N} / N \rightarrow \mathbb{R}, \quad G_{N}(0)=G_{N}(1)=0, \quad \forall N \in \mathbb{N},
$$

such that

$$
\sup _{x \in \widehat{\Lambda}_{N}}\left|G_{N}\left(\frac{x}{N}\right)-G\left(\frac{x}{N}\right)\right| \underset{N \rightarrow \infty}{\longrightarrow} 0 \quad \text { and } \quad \sup _{x \in \widehat{\Lambda}_{N}}\left|\mathcal{A}^{N} G_{N}\left(\frac{x}{N}\right)-\mathcal{A} G\left(\frac{x}{N}\right)\right| \underset{N \rightarrow \infty}{\longrightarrow} 0
$$

hold. 
Proof. The function $G_{N}$ is given as follows:

$$
G_{N}\left(\frac{x}{N}\right):=1_{\{x>0\}}\left\{\begin{array}{l}
\frac{\alpha}{N^{-\beta} \alpha_{L}}\left(G\left(\frac{1}{N}\right)-\frac{C_{N}(G)}{N}\right) \\
+\mathbf{1}_{\{1<x<N\}}\left(G\left(\frac{x}{N}\right)-G\left(\frac{1}{N}\right)-\frac{x-1}{N} C_{N}(G)\right) \\
+\mathbf{1}_{\{x=N\}}\left(G\left(\frac{N-1}{N}\right)-G\left(\frac{1}{N}\right)-\frac{N-2}{N} C_{N}(G)+\frac{\alpha}{N^{-\beta} \alpha_{R}}\left(-G\left(\frac{N-1}{N}\right)-\frac{C_{N}(G)}{N}\right)\right)
\end{array}\right\}
$$

where

$$
C_{N}(G):=\frac{\left(\frac{\alpha}{N^{-\beta} \alpha_{L}}-1\right) G\left(\frac{1}{N}\right)+G\left(\frac{N-1}{N}\right)\left(1-\frac{\alpha}{N^{-\beta} \alpha_{R}}\right)}{\frac{1}{N}\left(\frac{\alpha}{N^{-\beta} \alpha_{L}}+(N-2)+\frac{\alpha}{N^{-\beta} \alpha_{R}}\right)}
$$

is chosen such that $G_{N}(1)=0$. By applying the generator $A^{N}$ to such function, we obtain

$$
A^{N} G_{N}\left(\frac{x}{N}\right)=N^{2} \alpha\left(G\left(\frac{x+1}{N}\right)+G\left(\frac{x-1}{N}\right)-2 G\left(\frac{x}{N}\right)\right)=\alpha \Delta_{N} G\left(\frac{x}{N}\right)
$$

if $x \in \Lambda_{N}$ and $A^{N} G_{N}(0)=A^{N} G_{N}(1)=0$. As a consequence, we get the second convergence in 4.23. On the other side,

$$
\sup _{x \in \widehat{\Lambda}_{N}}\left|G_{N}\left(\frac{x}{N}\right)-G\left(\frac{x}{N}\right)\right| \leq\left|G\left(\frac{1}{N}\right)\right|\left(1+\frac{\alpha}{N^{-\beta} \alpha_{L}}\right)+\left|G\left(\frac{N-1}{N}\right)\right|\left(1+\frac{\alpha}{N^{-\beta} \alpha_{R}}\right) .
$$

Let us observe that $\left|G\left(\frac{1}{N}\right)\right| \leq \frac{1}{N}\left|\partial_{u}^{+} G(0)\right|+\frac{C_{0}}{N^{2}}$ and $\left|G\left(\frac{N-1}{N}\right)\right| \leq \frac{1}{N}\left|\partial_{u}^{-} G(1)\right|+\frac{C_{1}}{N^{2}}$ for all $N \in \mathbb{N}$ large enough and some constants $C_{0}, C_{1}>0$ independent of $N \in \mathbb{N}$. As a consequence, because $\beta \in[0,1)$, we obtain the first convergence in 4.23 . This concludes the proof.

Let us now prove convergence in probability of one-dimensional distributions, and notice that, by a union bound, the latter immediately yields convergence of finite-dimensional distributions. More precisely, we prove that, for all $\beta \in[0,1), G \in \mathscr{S}, t \geq 0$ and $\delta>0$, we have

$$
\mathbb{P}_{\mu^{N}}^{N}\left(\left|\left\langle G, \mathscr{Z}_{t}^{N}\right\rangle-\int_{0}^{t}\left\langle\mathcal{A} G, \mathscr{Z}_{s}^{N}\right\rangle \mathrm{d} s-\left\langle G, \vartheta_{0}-h_{\vartheta_{L}, \vartheta_{R}} \mathrm{~d} u\right\rangle\right|>\delta\right) \underset{N \rightarrow \infty}{\longrightarrow} 0 .
$$

By the triangle inequality, the above follows if we can show that, for all $\delta>0$,

$$
\mathbb{P}_{\mu^{N}}^{N}\left(\left|\left\langle G, \mathscr{Z}_{t}^{N}\right\rangle-\left\langle G_{N}, \mathscr{Z}_{t}^{N}\right\rangle\right|>\delta\right) \underset{N \rightarrow \infty}{\longrightarrow} 0
$$

and

$$
\mathbb{P}_{\mu^{N}}^{N}\left(\left|\left\langle G_{N}, \mathscr{Z}_{t}^{N}\right\rangle-\int_{0}^{t}\left\langle\mathcal{A} G, \mathscr{Z}_{s}^{N}\right\rangle \mathrm{d} s-\left\langle G, \vartheta_{0}-h_{\vartheta_{L}, \vartheta_{R}} \mathrm{~d} u\right\rangle\right|>\delta\right) \underset{N \rightarrow \infty}{\longrightarrow} 0
$$

hold, where the functions $\left\{G_{N}: N \in \mathbb{N}\right\}$ are those given in Lemma 4.2. The claim in 4.26 follows at once from Hölder's inequality, the uniform bounds in 4.1 and Lemma 4.2.

Let us now deal with the claim in 4.27) by means of Dynkin's formula for $\left\{\left\langle G_{N}, \mathscr{Z}^{N}\right\rangle: N \in \mathbb{N}\right\}$. As a first step, we have

$$
\begin{aligned}
& \mathbb{P}_{\mu^{N}}^{N}\left(\mid\left\langle G_{N}, \mathscr{Z}_{0}^{N}\right\rangle-\left\langle G,\left(\vartheta_{0}-h_{\vartheta_{L}, \vartheta_{R}} \mathrm{~d} u\right) \alpha\right|>\delta\right) \\
\leq & \mathbb{P}_{\mu^{N}}^{N^{N}}\left(\left|\left\langle G_{N}, \mathscr{Z}_{0}^{N}\right\rangle-\left\langle G, \mathscr{Z}_{0}^{N}\right\rangle\right|>\frac{\delta}{2}\right) \\
+ & \mathbb{P}_{\mu^{N}}^{N}\left(\mid\left\langle G, \mathscr{Z}_{0}^{N}\right\rangle-\left\langle G,\left(\vartheta_{0}-h_{\vartheta_{L}, \vartheta_{R}} \mathrm{~d} u\right) \alpha\right|>\frac{\delta}{2}\right)
\end{aligned}
$$

and both terms on the r.h.s. vanish as $N \rightarrow \infty$; more specifically, the first term vanishes because of Markov's inequality, assumption (b) and the first convergence in (4.23), while the second term because of assumption (a) and Lemma 4.1. Moreover, for all $\delta>0$ and $t \geq 0$, we have

$$
\mathbb{P}_{\mu^{N}}^{N}\left(\left|\int_{0}^{t}\left(\mathcal{L}^{N}\left\langle G_{N}, \mathscr{Z}_{s}^{N}\right\rangle-\left\langle\mathcal{A} G, \mathscr{Z}_{s}^{N}\right\rangle\right) \mathrm{d} s\right|>\delta\right) \underset{N \rightarrow \infty}{\longrightarrow} 0
$$


which follows by Markov's inequality, duality (4.3), the symmetry of $A^{N}$ as in 4.6), Tonelli's theorem, 4.1) and the second convergence in 4.23):

$$
\begin{aligned}
& \mathbb{P}_{\mu^{N}}^{N}\left(\left|\int_{0}^{t}\left(\mathcal{L}^{N}\left\langle G_{N}, \mathscr{Z}_{s}^{N}\right\rangle-\left\langle\mathcal{A} G, \mathscr{Z}_{s}^{N}\right\rangle\right) \mathrm{d} s\right|>\delta\right) \\
\leq & \frac{1}{\delta} \mathbb{E}_{\mu^{N}}^{N}\left[\int_{0}^{t} \frac{1}{N} \sum_{x \in \Lambda_{N}}\left|\mathcal{A}^{N} G_{N}\left(\frac{x}{N}\right)-\mathcal{A} G\left(\frac{x}{N}\right)\right|\left(D_{N}\left(x, \eta_{s}^{N}\right)+h_{\vartheta_{L}, \vartheta_{R}}^{N}(x)\right) \alpha \mathrm{d} s\right] \\
\leq & \frac{t}{\delta} \sup _{x \in \widehat{\Lambda}_{N}}\left|\mathcal{A}^{N} G_{N}\left(\frac{x}{N}\right)-\mathcal{A} G\left(\frac{x}{N}\right)\right|\left\{\sup _{s \in[0, t]} \mathbb{E}_{\mu^{N}}^{N}\left[D_{N}\left(x, \eta_{s}^{N}\right)\right]+\max \left\{\vartheta_{L}, \vartheta_{R}\right\}\right\} .
\end{aligned}
$$

In conclusion, the martingales arising from Dynkin's decomposition of $\left\{\left\langle G_{N}, \mathscr{Z}^{N}\right\rangle: N \in \mathbb{N}\right\}$ vanish in probability as $N \rightarrow \infty$. Indeed, for all $t \geq 0$,

$$
\mathbb{E}_{\mu^{N}}^{N}\left[\left(\left\langle G_{N}, \mathscr{M}_{t}^{N}\right\rangle\right)^{2}\right] \leq \frac{C t}{N}\left\{\frac{1}{N} \sum_{x \in \Lambda_{N}}\left(-\mathcal{A}^{N} G_{N}\left(\frac{x}{N}\right)\right) G_{N}\left(\frac{x}{N}\right)\right\} \underset{N \rightarrow \infty}{\longrightarrow} 0
$$

because the expression between curly brackets is uniformly bounded in $N \rightarrow \infty$ by (4.23) and where, by (4.1), $C=C\left(\kappa, \vartheta_{L}, \vartheta_{R}\right)>0$ is a constant independent of $N \in \mathbb{N}$ and $t \geq 0$.

The proof of Theorem 3.1 for the case $\beta \in[0,1)$ ends as soon as we show, by Mitoma's tightness criterion [41, that, for all $G \in \mathscr{S}$, the sequence $\left\{\left\langle G, \mathscr{Z}^{N}\right\rangle: N \in \mathbb{N}\right\}$ is tight in $\mathcal{D}([0, \infty), \mathbb{R})$. Most of the steps of this proof may be adapted from those in Section 4.1.1. with the only exceptions that, for all $G \in \mathscr{S}$ and $t \geq 0$, the following boundary terms

$$
\begin{gathered}
\mathbb{E}_{\mu^{N}}^{N}\left[\left(\alpha \nabla_{N}^{+} G(0)-\alpha_{L} N^{1-\beta} G\left(\frac{1}{N}\right)\right)^{2}\left(D_{N}\left(1, \eta_{t}^{N}\right)-h_{\vartheta_{L}, \vartheta_{R}}^{N}(1)\right)^{2} \alpha^{2}\right] \\
\mathbb{E}_{\mu^{N}}^{N}\left[\left(\alpha \nabla_{N}^{-} G(1)-\alpha_{R} N^{1-\beta} G\left(\frac{N-1}{N}\right)\right)^{2}\left(D_{N}\left(N-1, \eta_{t}^{N}\right)-h_{\vartheta_{L}, \vartheta_{R}}^{N}(N-1)\right)^{2} \alpha^{2}\right]
\end{gathered}
$$

and

$$
\frac{1}{N^{2}}\left\{N^{2-\beta} \alpha_{L}\left(G\left(\frac{1}{N}\right)\right)^{2} \mathbb{E}_{\mu^{N}}^{N}\left[\mathcal{V}_{\{0,1\}}^{N}\left(\eta_{t}^{N}\right)\right] \alpha+N^{2-\beta} \alpha_{R}\left(G\left(\frac{N-1}{N}\right)\right)^{2} \mathbb{E}_{\mu^{N}}^{N}\left[\mathcal{V}_{\{N-1, N\}}^{N}\left(\eta_{t}^{N}\right)\right] \alpha\right\}
$$

are uniformly bounded in $N \in \mathbb{N}$ because of the boundary conditions 2.17) that $G \in \mathscr{S}$ satisfies and the uniform bounds in 4.1 .

4.1.3. Some considerations for the case $\beta<0$. The particle system dynamics described by the generator $\mathcal{L}^{N}$ in (2.1) as well as the duality relations and the results in Lemmas 4.1 and 4.2 clearly extend to the setting of "fast" boundary, i.e., $\beta<0$ if constructing $\mathscr{S}$ for $\beta<0$ as done for the case $\beta \in[0,1)$. Moreover, from the first part of the proof in Section 4.1.2, it follows that, for all $\beta<0$ and $G \in \mathscr{S}$, the sequence

$$
\left\{\left\langle G_{N}, \mathscr{Z}_{.}^{N}\right\rangle: N \in \mathbb{N}\right\}
$$

is tight in $\mathcal{D}([0, \infty), \mathbb{R})$, where the sequence $\left\{G_{N}: N \in \mathbb{N}\right\}$ is the one given in Lemma 4.2, and, for all $T>0$ and $\delta>0$, the following convergence

$$
\mathbb{P}_{\mu^{N}}^{N}\left(\sup _{t \in[0, T]}\left|\left\langle G_{N}, \mathscr{Z}_{t}^{N}\right\rangle-\left\langle G,\left(\vartheta(t)-h_{\vartheta_{L}, \vartheta_{R}} \mathrm{~d} u\right) \alpha\right\rangle\right|>\delta\right) \underset{N \rightarrow \infty}{\longrightarrow} 0
$$

holds, where $\left\{\vartheta(t)-h_{\vartheta_{L}, \vartheta_{R}} \mathrm{~d} u: t \geq 0\right\} \in \mathcal{C}\left([0, \infty), \mathscr{S}^{\prime}\right)$ is the unique Dirichlet solution in $\mathscr{S}^{\prime}$ with initial condition given by $\vartheta_{0}-h_{\vartheta_{L}, \vartheta_{R}} \mathrm{~d} u$. Moreover, by Lemma 4.2 and the uniform bounds in 4.1., it follows that, for all $\beta<0, G \in \mathscr{S}, t \geq 0$ and $\delta>0$,

$$
\mathbb{P}_{\mu^{N}}^{N}\left(\left|\left\langle G, \mathscr{Z}_{t}^{N}\right\rangle-\left\langle G_{N}, \mathscr{Z}_{t}^{N}\right\rangle\right|>\delta\right) \underset{N \rightarrow \infty}{\longrightarrow} 0
$$


yielding, in particular, convergence of the finite dimensional distribution for the fields $\left\{\mathscr{Z}^{N}: N \in \mathbb{N}\right\}$ : for all $\beta<0, G \in \mathscr{S}, t \geq 0$ and $\delta>0$,

$$
\mathbb{P}_{\mu^{N}}^{N}\left(\left|\left\langle G, \mathscr{Z}_{t}^{N}\right\rangle-\left\langle G,\left(\vartheta(t)-h_{\vartheta_{L}, \vartheta_{R}} \mathrm{~d} u\right) \alpha\right\rangle\right|>\delta\right) \underset{N \rightarrow \infty}{\longrightarrow} 0 .
$$

However, tightness of the empirical density fields $\left\{\mathscr{Z}^{N}: N \in \mathbb{N}\right\}$ in $\mathcal{D}\left([0, \infty), \mathscr{S}^{\prime}\right)$ for the case $\beta<0$ does not follow from the arguments used in the second part of Section 4.1 .2 above because the boundary terms in 4.29 and 4.30 are not, in general, uniformly bounded in $N \in \mathbb{N}$.

An alternative approach to derive the hydrodynamic limit for the case $\beta<0$ would be, in view of 4.31, to strengthen the convergence in 4.32 by requiring, for all $T>0$ and $\delta>0$,

$$
\mathbb{P}_{\mu^{N}}^{N}\left(\sup _{t \in[0, T]}\left|\left\langle G, \mathscr{Z}_{t}^{N}\right\rangle-\left\langle G_{N}, \mathscr{Z}_{t}^{N}\right\rangle\right|>\delta\right) \underset{N \rightarrow \infty}{\longrightarrow} 0
$$

Because of Lemma 4.2 (4.34 would follow, by Markov's inequality, from

$$
\sup _{N \in \mathbb{N}} \mathbb{E}_{\mu^{N}}^{N}\left[\sup _{t \in[0, T]}\left(\frac{1}{N} \sum_{x \in \Lambda_{N}} \eta_{t}^{N}(x)\right)^{p}\right]<\infty
$$

for some $p>0$. However, while 4.35) is trivially satisfied by the SIP for which each site can accommodate at most one particle at the time, this is no more the case for the open SIP and the validity of 4.35 is not guaranteed.

4.2. Proof of Theorem 3.2. We split the proof of Theorem 3.2 in two parts: we first show that assumption (b) and then that assumption (a) of Theorem 3.1 hold for the sequence $\left\{\mu_{\vartheta_{L}, \vartheta_{R}}^{N}: N \in \mathbb{N}\right\}$. Once these assumptions are verified, Theorem 3.1 applies, yielding the hydrostatic limit. We remark that the arguments employed in this section hold true also for negative values of the parameter $\beta$.

Let us introduce, for all $\beta \geq 0$ and $N \in \mathbb{N}$, the following function

$$
k_{\vartheta_{L}, \vartheta_{R}}^{N}(x, y):=E_{\mu_{\vartheta_{L}, \vartheta_{R}}^{N}}\left[D_{N}(x, y, \eta)\right]=\lim _{t \rightarrow \infty} \mathbb{E}_{\mu^{N}}^{N}\left[D_{N}\left(x, y, \eta_{t}^{N}\right)\right], \quad x, y \in \widehat{\Lambda}_{N}
$$

for any probability measure $\mu^{N}$ on $\mathcal{X}_{N}$, which, by stationarity of $\mu_{\vartheta_{L}, \vartheta_{R}}^{N}$ and duality $[2.13$, solves the following linear boundary value problem:

$$
\left\{\begin{aligned}
B^{N} f(x, y) & =0 & & (x, y) \in \Lambda_{N} \times \Lambda_{N} \\
f(x, y) & =h_{\vartheta_{L}, \vartheta_{R}}^{N}(x) h_{\vartheta_{L}, \vartheta_{R}}^{N}(y) & & (x, y) \in\left(\widehat{\Lambda}_{N} \times \widehat{\Lambda}_{N}\right) \backslash\left(\Lambda_{N} \times \Lambda_{N}\right),
\end{aligned}\right.
$$

where we recall that $B^{N}$ is the infinitesimal generator corresponding to two inclusion particles in $\Lambda_{N}$ with absorbing sites $\{0, N\}$ as defined in 2.14 and $h_{\vartheta_{L}, \vartheta_{R}}^{N}$ is the solution of (4.9) and given in 4.12. We note that, while for the open symmetric exclusion process the stationary two-point correlations are known (see, e.g., [14, [30, Eq. (2.23)]), for the open symmetric inclusion process the function $k_{\vartheta_{L}, \vartheta_{R}}^{N}$ is not, in general, explicit.

4.2.1. Assumption (b) of Theorem 3.1 for the stationary measure. By the maximum principle applied to the boundary value problems (4.9) and (4.37), we obtain

$$
0 \leq h_{\vartheta_{L}, \vartheta_{R}}^{N}(x) \leq \max \left\{\vartheta_{L}, \vartheta_{R}\right\} \quad \text { and } \quad 0 \leq k_{\vartheta_{L}, \vartheta_{R}}^{N}(x, y) \leq \max \left\{\vartheta_{L}^{2}, \vartheta_{R}^{2}\right\}
$$

for all $x, y \in \widehat{\Lambda}_{N}$, yielding, by (4.8) and (4.36), the bounds in assumption (b) of Theorem 3.1 with $\kappa=\max \left\{\vartheta_{L}, \vartheta_{R}\right\}$. 
4.2.2. Assumption (a) of Theorem 3.1 for the stationary measure. In this section we prove that, for all $\beta \geq 0, G \in \mathscr{S}$ and $\delta>0$, we have

$$
\mu_{\vartheta_{L}, \vartheta_{R}}^{N}\left(\left\{\eta \in \mathcal{X}_{N}:\left|\frac{1}{N} \sum_{x \in \Lambda_{N}} G\left(\frac{x}{N}\right) \eta(x)-\left\langle G, h_{\vartheta_{L}, \vartheta_{R}} \mathrm{~d} u \alpha\right\rangle\right|>\delta\right\}\right) \underset{N \rightarrow \infty}{\longrightarrow} 0
$$

or, equivalently by Lemma 4.1 .

$$
\mu_{\vartheta_{L}, \vartheta_{R}}^{N}\left(\left\{\eta \in \mathcal{X}_{N}:\left|\frac{1}{N} \sum_{x \in \Lambda_{N}} G\left(\frac{x}{N}\right)\left(D_{N}(x, \eta)-h_{\vartheta_{L}, \vartheta_{R}}^{N}(x)\right) \alpha\right|>\delta\right\}\right) \underset{N \rightarrow \infty}{\longrightarrow} 0,
$$

where $h_{\vartheta_{L}, \vartheta_{R}} \mathrm{~d} u \in \mathcal{C}^{\infty}([0,1])$ is given in either (3.8) if $\beta<1$, (3.9) if $\beta=1$ or (3.10) if $\beta>1$. In view of Chebyshev's inequality, we prove

$$
E_{\mu_{\vartheta_{L}, \vartheta_{R}}^{N}}\left[\left(\frac{1}{N} \sum_{x \in \Lambda_{N}} G\left(\frac{x}{N}\right)\left(D_{N}(x, \eta)-h_{\vartheta_{L}, \vartheta_{R}}^{N}(x)\right) \alpha\right)^{2}\right] \underset{N \rightarrow \infty}{\longrightarrow} 0,
$$

from which 4.39 follows for all $\delta>0$. To this purpose, by 4.16 and stationarity of $\mu_{\vartheta_{L}, \vartheta_{R}}^{N}$, we have

$$
\begin{aligned}
& E_{\mu_{\vartheta_{L}, \vartheta_{R}}^{N}}\left[\left(\frac{1}{N} \sum_{x \in \Lambda_{N}} G\left(\frac{x}{N}\right)\left(D_{N}(x, \eta)-h_{\vartheta_{L}, \vartheta_{R}}^{N}(x)\right) \alpha\right)^{2}\right] \\
= & \left\langle\left\langle G \otimes G, k_{\vartheta_{L}, \vartheta_{R}}^{N}\right\rangle_{N \times N}-\left(\left\langle G, h_{\vartheta_{L}, \vartheta_{R}}^{N}\right\rangle_{N}\right)^{2}+\frac{1}{N}\left\langle\left\langle G^{2}, h_{\vartheta_{L}, \vartheta_{R}}^{N}\right\rangle_{N}\right.\right. \\
= & \frac{1}{N^{2}} \sum_{x, y \in \Lambda_{N}} G\left(\frac{x}{N}\right) G\left(\frac{y}{N}\right) k_{\vartheta_{L}, \vartheta_{R}}^{N}(x, y) \alpha\left(\alpha+\mathbf{1}_{\{x=y\}}\right) \\
- & \frac{1}{N^{2}} \sum_{x, y \in \Lambda_{N}} G\left(\frac{x}{N}\right) G\left(\frac{y}{N}\right) h_{\vartheta_{L}, \vartheta_{R}}^{N}(x) h_{\vartheta_{L}, \vartheta_{R}}^{N}(y) \alpha^{2}+\frac{1}{N^{2}} \sum_{x \in \Lambda_{N}} G\left(\frac{x}{N}\right)^{2} h_{\vartheta_{L}, \vartheta_{R}}^{N}(x) \alpha \\
= & \frac{1}{N^{2}} \sum_{x, y \in \Lambda_{N}} G\left(\frac{x}{N}\right) G\left(\frac{y}{N}\right)\left(k_{\vartheta_{L}, \vartheta_{R}}^{N}(x, y)-h_{\vartheta_{L}, \vartheta_{R}}^{N}(x) h_{\vartheta_{L}, \vartheta_{R}}^{N}(y)\right) \alpha\left(\alpha+\mathbf{1}_{\{x=y\}}\right) \\
+ & \frac{1}{N}\left\{\frac{1}{N} \sum_{x \in \Lambda_{N}} G\left(\frac{x}{N}\right)^{2}\left(1+h_{\vartheta_{L}, \vartheta_{R}}^{N}(x)\right) h_{\vartheta_{L}, \vartheta_{R}}^{N}(x) \alpha\right\} .
\end{aligned}
$$

By the uniform boundedness of $G \in \mathscr{S}$ and $\left\{h_{\vartheta_{L}, \vartheta_{R}}^{N}: N \in \mathbb{N}\right\}$, the second term on the r.h.s. above vanishes as $N \rightarrow \infty$. Hence, we are left only with the proof that

$$
\begin{aligned}
& \left\langle\left\langle G \otimes G, k_{\vartheta_{L}, \vartheta_{R}}^{N}-h_{\vartheta_{L}, \vartheta_{R}}^{N} \otimes h_{\vartheta_{L}, \vartheta_{R}}^{N}\right\rangle_{N \times N}\right. \\
= & \frac{1}{N^{2}} \sum_{x, y \in \Lambda_{N}} G\left(\frac{x}{N}\right) G\left(\frac{y}{N}\right)\left(k_{\vartheta_{L}, \vartheta_{R}}^{N}(x, y)-h_{\vartheta_{L}, \vartheta_{R}}^{N}(x) h_{\vartheta_{L}, \vartheta_{R}}^{N}(y)\right) \alpha\left(\alpha+1_{\{x=y\}}\right) \underset{N \rightarrow \infty}{\longrightarrow} 0 .
\end{aligned}
$$

More specifically, we obtain 4.41 from the following upper bound: for all $\beta \geq 0$ and $G \in \mathscr{S}$, we have

$$
\begin{aligned}
& \sup _{N \in \mathbb{N}} \max \left\{N, N^{\beta-1}\right\}\left|\left\langle G \otimes G, k_{\vartheta_{L}, \vartheta_{R}}^{N}-h_{\vartheta_{L}, \vartheta_{R}}^{N} \otimes h_{\vartheta_{L}, \vartheta_{R}}^{N}\right\rangle_{N \times N}\right| \\
\leq & \sup _{N \in \mathbb{N}} \max \left\{N, N^{\beta-1}\right\}\|G \otimes G\|_{\infty}\left\langle\left\langle 1 \otimes 1,\left|k_{\vartheta_{L}, \vartheta_{R}}^{N}-h_{\vartheta_{L}, \vartheta_{R}}^{N} \otimes h_{\vartheta_{L}, \vartheta_{R}}^{N}\right|\right\rangle_{N \times N}<\infty .\right.
\end{aligned}
$$

We remark that the upper bound in 4.42 differs from those in, e.g., [37, Eq. (3.2)] and [30, Proposition 2.1] derived for the open symmetric exclusion process from the explicit expression of the two-point stationary correlation function and corresponding, in our setting, to

$$
\sup _{N \in \mathbb{N}} \max \left\{N, N^{\beta-1}\right\}\left|\left\langle G \otimes G, k_{\vartheta_{L}, \vartheta_{R}}^{N}-h_{\vartheta_{L}, \vartheta_{R}}^{N} \otimes h_{\vartheta_{L}, \vartheta_{R}}^{N}\right\rangle_{N \times N}\right|
$$




$$
\begin{aligned}
& \leq \sup _{N \in \mathbb{N}} \max \left\{N, N^{\beta-1}\right\}\langle\langle|G \otimes G|, 1 \otimes 1\rangle\rangle_{N \times N} \sup _{(x, y) \in \Lambda_{N} \times \Lambda_{N}}\left|k_{\vartheta_{L}, \vartheta_{R}}^{N}(x, y)-h_{\vartheta_{L}, \vartheta_{R}}^{N}(x) h_{\vartheta_{L}, \vartheta_{R}}^{N}(y)\right| \\
& <\infty
\end{aligned}
$$

In our case, although we do not know, as already mentioned above, the explicit form of

$$
k_{\vartheta_{L}, \vartheta_{R}}^{N}(x, y)-h_{\vartheta_{L}, \vartheta_{R}}^{N}(x) h_{\vartheta_{L}, \vartheta_{R}}^{N}(y), \quad x, y \in \Lambda_{N}
$$

by [23, Theorem 3.4] and [23, Lemma 3.5] (see also [23, Remark 3.6(b)]), we know the sign of these stationary two-point correlation functions as well as the following representation in terms of absorption probabilities of two inclusion particles. Recalling the notation in Section 2.2.1, we have

$$
k_{\vartheta_{L}, \vartheta_{R}}^{N}(x, y)-h_{\vartheta_{L}, \vartheta_{R}}^{N}(x) h_{\vartheta_{L}, \vartheta_{R}}^{N}(y)>0
$$

and

$$
\begin{aligned}
& k_{\vartheta_{L}, \vartheta_{R}}^{N}(x, y)-h_{\vartheta_{L}, \vartheta_{R}}^{N}(x) h_{\vartheta_{L}, \vartheta_{R}}^{N}(y) \\
= & \int_{0}^{\infty} \sum_{\substack{z \in \Lambda_{N} \\
z \neq N-1}}^{\infty}\left\{\begin{array}{c}
N^{2}\left(h_{\vartheta_{L}, \vartheta_{R}}^{N}(z+1)-h_{\vartheta_{L}, \vartheta_{R}}^{N}(z)\right)^{2} \\
\times \widehat{\mathbb{P}}_{\xi=\delta_{x}+\delta_{y}}^{N}\left(\xi_{s}^{N}(z)=1 \text { and } \xi_{s}^{N}(z+1)=1\right)
\end{array}\right\} \mathrm{d} s
\end{aligned}
$$

for all $x, y \in \Lambda_{N}$.

Remark 4.1. The above expression for the stationary two-point correlations is related to the stationary solution to the non-homogeneous parabolic difference system in Eqs. (2.13)-(2.15) in [30. (see also [37]). However, we remark that, while the solution in [30] is obtained by means of Duhamel's principle in terms of the Markov semigroup of two independent random walks, the identity (4.44) is obtained by solving a linear system of evolution equations involving second order duality functions and the Markov semigroup of two interacting dual inclusion particles. The representation of the solution in terms of such Markov semigroup - symmetric with respect to $\left\langle\langle\cdot, \cdot\rangle_{N \times N}\right.$ for functions vanishing at the boundary - will turn out useful later on.

As a consequence of 4.43, we get

$$
\begin{aligned}
& \left|\frac{1}{N^{2}} \sum_{x, y \in \Lambda_{N}} G\left(\frac{x}{N}\right) G\left(\frac{y}{N}\right)\left(k_{\vartheta_{L}, \vartheta_{R}}^{N}(x, y)-h_{\vartheta_{L}, \vartheta_{R}}^{N}(x) h_{\vartheta_{L}, \vartheta_{R}}^{N}(y)\right) \alpha\left(\alpha+\mathbf{1}_{\{x=y\}}\right)\right| \\
\leq & \|G\|_{\infty}^{2} \frac{1}{N^{2}} \sum_{x, y \in \Lambda_{N}}\left(k_{\vartheta_{L}, \vartheta_{R}}^{N}(x, y)-h_{\vartheta_{L}, \vartheta_{R}}^{N}(x) h_{\vartheta_{L}, \vartheta_{R}}^{N}(y)\right) \alpha\left(\alpha+\mathbf{1}_{\{x=y\}}\right) .
\end{aligned}
$$

while, by 4.12 and

$$
N^{2}\left(h_{\vartheta_{L}, \vartheta_{R}}^{N}(z+1)-h_{\vartheta_{L}, \vartheta_{R}}^{N}(z)\right)^{2} \leq \frac{C\left(\vartheta_{L}-\vartheta_{R}\right)^{2}}{\max \left\{1, N^{2 \beta-2}\right\}}, \quad z \in \Lambda_{N} \backslash\{N-1\},
$$

for some constant $C=C\left(\alpha, \alpha_{L}, \alpha_{R}\right)>0$, we further obtain

$$
\begin{aligned}
& \frac{1}{N^{2}} \sum_{x, y \in \Lambda_{N}}\left(k_{\vartheta_{L}, \vartheta_{R}}^{N}(x, y)-h_{\vartheta_{L}, \vartheta_{R}}^{N}(x) h_{\vartheta_{L}, \vartheta_{R}}^{N}(y)\right) \alpha\left(\alpha+\mathbf{1}_{\{x=y\}}\right) \\
\leq & \frac{C\left(\vartheta_{L}-\vartheta_{R}\right)^{2}}{\max \left\{1, N^{2 \beta-2}\right\}} \frac{1}{N^{2}} \sum_{x, y \in \Lambda_{N}} \int_{0}^{\infty} \sum_{\substack{z \in \Lambda_{N} \\
z \neq N-1}} \widehat{\mathbb{P}}_{\xi=\delta_{x}+\delta_{y}}^{N}\left(\xi_{s}^{N}(z)=1 \text { and } \xi_{s}^{N}(z+1)=1\right) \alpha\left(\alpha+\mathbf{1}_{\{x=y\}}\right) \mathrm{d} s \\
= & \frac{C\left(\vartheta_{L}-\vartheta_{R}\right)^{2}}{\max \left\{1, N^{2 \beta-2}\right\}} \frac{1}{N^{2}} \sum_{x, y \in \Lambda_{N}} \int_{0}^{\infty} \mathrm{P}^{N}\left(\begin{array}{c}
\left(X_{s}^{N, x}, Y_{s}^{N, y}\right) \in \Lambda_{N} \times \Lambda_{N} \\
\text { and }\left|X_{s}^{N, x}-Y_{s}^{N, y}\right|=1
\end{array}\right) \alpha\left(\alpha+\mathbf{1}_{\{x=y\}}\right) \mathrm{d} s,
\end{aligned}
$$

where in the last step we went from an unlabeled to a labeled representation of the dual system consisting of two inclusion particles evolving according to the infinitesimal generator $B^{N}$ given in 
(2.14) and with $\mathrm{P}^{N}$, resp. $\mathrm{E}^{N}$, denoting the corresponding law, resp. expectation: for all $(x, y) \in \widehat{\Lambda}_{N} \times \widehat{\Lambda}_{N}$,

$$
\left\{\left(X_{t}^{N, x}, Y_{t}^{N, y}\right): t \geq 0\right\} \subset \widehat{\Lambda}_{N} \times \widehat{\Lambda}_{N}
$$

denotes the Markov process with generator $B^{N}$ and initial conditions given by

$$
\left(X_{0}^{N, x}, Y_{0}^{N, y}\right)=(x, y) \quad \text { a.s. . }
$$

If we let $\left\{S_{t}^{N}: t \geq 0\right\}$ denote the Markov semigroup associated with the generator $B^{N}$, then

$$
\begin{aligned}
& \frac{C\left(\vartheta_{L}-\vartheta_{R}\right)^{2}}{\max \left\{1, N^{2 \beta-2}\right\}} \frac{1}{N^{2}} \sum_{x, y \in \Lambda_{N}} \int_{0}^{\infty} \mathrm{P}^{N}\left(\begin{array}{c}
\left(X_{s}^{N, x}, Y_{s}^{N, y}\right) \in \Lambda_{N} \times \Lambda_{N} \\
\text { and }\left|X_{s}^{N, x}-Y_{s}^{N, y}\right|=1
\end{array}\right) \alpha\left(\alpha+\mathbf{1}_{\{x=y\}}\right) \mathrm{d} s \\
= & \frac{C\left(\vartheta_{L}-\vartheta_{R}\right)^{2}}{\max \left\{1, N^{2 \beta-2}\right\}} \frac{1}{N^{2}} \sum_{x, y \in \Lambda_{N}} \int_{0}^{\infty} S_{s}^{N} f_{N}(x, y) \alpha\left(\alpha+\mathbf{1}_{\{x=y\}}\right) \mathrm{d} s
\end{aligned}
$$

where the function $f_{N}: \widehat{\Lambda}_{N} \times \widehat{\Lambda}_{N} \rightarrow \mathbb{R}$ is defined as follows:

$$
f_{N}(x, y):= \begin{cases}1 & \text { if } x, y \in \Lambda_{N} \text { and }|x-y|=1 \\ 0 & \text { otherwise }\end{cases}
$$

Moreover, by Tonelli's theorem and by the symmetry of $B^{N}$ - and, consequently, of the corresponding semigroup - with respect to the inner product $\left\langle\langle\cdot, \cdot\rangle_{N \times N}\right.$ for functions vanishing on $\left(\widehat{\Lambda}_{N} \times \widehat{\Lambda}_{N}\right) \backslash\left(\Lambda_{N} \times \Lambda_{N}\right)(\operatorname{cf} .(2.16))$, we obtain

$$
\begin{aligned}
& \frac{C\left(\vartheta_{L}-\vartheta_{R}\right)^{2}}{\max \left\{1, N^{2 \beta-2}\right\}} \frac{1}{N^{2}} \sum_{x, y \in \Lambda_{N}} \int_{0}^{\infty} S_{s}^{N} f_{N}(x, y) \alpha\left(\alpha+\mathbf{1}_{\{x=y\}}\right) \mathrm{d} s \\
= & \frac{C\left(\vartheta_{L}-\vartheta_{R}\right)^{2}}{\max \left\{1, N^{2 \beta-2}\right\}} \frac{1}{N^{2}} \sum_{x, y \in \Lambda_{N}} \int_{0}^{\infty} f_{N}(x, y)\left(S_{s}^{N} g_{N}(x, y)\right) \alpha\left(\alpha+\mathbf{1}_{\{x=y\}}\right) \mathrm{d} s \\
= & \frac{C\left(\vartheta_{L}-\vartheta_{R}\right)^{2}}{\max \left\{1, N^{2 \beta-2}\right\}} \int_{0}^{\infty}\left\langle\left\langle f_{N}, S_{s}^{N} g_{N}\right\rangle_{N \times N} \mathrm{~d} s,\right.
\end{aligned}
$$

where the function $g_{N}: \widehat{\Lambda}_{N} \times \widehat{\Lambda}_{N} \rightarrow \mathbb{R}$ is the indicator function on $\Lambda_{N} \times \Lambda_{N} \subset \widehat{\Lambda}_{N} \times \widehat{\Lambda}_{N}$ :

$$
g_{N}(x, y):=\mathbf{1}_{\left\{(x, y) \in \Lambda_{N} \times \Lambda_{N}\right\}} .
$$

By Hölder's inequality, we have

$$
\left\langle\left\langle f_{N}, S_{s}^{N} g_{N}\right\rangle_{N \times N} \leq\left\langle\left\langle f_{N}, 1\right\rangle\right\rangle_{N \times N} \sup _{x, y \in \Lambda_{N}} S_{s}^{N} g_{N}(x, y)\right.
$$

(all functions are non-negative) and

$$
\left\langle\left\langle f_{N}, 1\right\rangle\right\rangle_{N \times N}=\frac{1}{N^{2}} \sum_{x, y \in \Lambda_{N}} \mathbf{1}_{\{|x-y|=1\}} \alpha\left(\alpha+\mathbf{1}_{\{x=y\}}\right) \leq \frac{2 \alpha^{2}}{N} .
$$

As a consequence, we further get

$$
\mid \frac{C\left(\vartheta_{L}-\vartheta_{R}\right)^{2}}{\max \left\{1, N^{2 \beta-2}\right\}} \int_{0}^{\infty}\left\langle\left\langle f_{N}, S_{s}^{N} g_{N}\right\rangle_{N \times N} \mathrm{~d} s\right| \leq \frac{2 \alpha^{2} C\left(\vartheta_{L}-\vartheta_{R}\right)^{2}}{\max \left\{N, N^{2 \beta-1}\right\}} \sup _{x, y \in \Lambda_{N}} \int_{0}^{\infty} S_{s}^{N} g_{N}(x, y) \mathrm{d} s .
$$

The proof to show that assumption (a) of Theorem 3.1 holds for the stationary measures ends if we can show that the r.h.s. above vanishes as $N \rightarrow \infty$. This last result is the content of the following lemma, whose proof is based on two main ingredients: first, by switching to the system 
of two inclusion particles to a suitable system of two "hierarchical" first and second class inclusion particles, we provide an upper bound for

$$
\sup _{x, y \in \Lambda_{N}} \int_{0}^{\infty} S_{s}^{N} g_{N}(x, y) \mathrm{d} s
$$

in terms of an expression involving only the absorption probabilities for a single non-interacting particle; then, we conclude by employing the asymptotic result in Lemma B.1 below on the absorption probability of the random walk with generator $A^{N}$ defined in $(2.10)$.

Lemma 4.3. For all $\beta \geq 0$,

$$
\sup _{N \in \mathbb{N}} \frac{1}{\max \left\{1, N^{\beta-1}\right\}} \sup _{x, y \in \Lambda_{N}} \int_{0}^{\infty} S_{s}^{N} g_{N}(x, y) \mathrm{d} s<\infty .
$$

As a consequence, for all $\beta \geq 0$ and $G \in \mathscr{S}$,

$$
\limsup _{N \rightarrow \infty} \max \left\{N, N^{\beta}\right\}\left|\left\langle G \otimes G, k_{\vartheta_{L}, \vartheta_{R}}^{N}-h_{\vartheta_{L}, \vartheta_{R}}^{N} \otimes h_{\vartheta_{L}, \vartheta_{R}}^{N}\right\rangle_{N \times N}\right|<\infty .
$$

We present the proof of Lemma 4.3 in the Section 4.2 .3 , in which we also introduce the notion of first and second class inclusion particles.

4.2.3. First 83 second class inclusion particles and Proof of Lemma 4.3. As for the SEP there is a well-known notion of first class and second class particles (see, e.g., 39, Part III, p. 218]), we show that an analogue notion exists for the SIP Roughly speaking, first class particles in the exclusion process evolve regardless of the positions of second class particles and, if their decision is to jump on a site occupied by a second class particle, the latter is "forced" to leave its place and occupy the place left vacant by the first class particle. In particular, the first class particle evolves as a non-interacting random walk, while the second class particle evolves as an interacting random walk.

Inspired by lookdown constructions available for population genetics models (see, e.g., [15]), a similar picture holds for the SIP. Indeed, while the first class inclusion particle evolves as a noninteracting random walk, the dynamics of the second class inclusion particle is determined by the superposition of two distinct effects: on the first place, it performs non-interacting random walk jumps and, on the second place, it "looks down" to the first class particle and "joins" it at rate two if the latter sits at a nearest-neighboring site. In Proposition 4.1 we show that, up to average over the role of first and second class particles at time $t=0$, the distribution at any later time $t>0$ of an unlabeled hierarchical "lookdown" process coincides with that of an unlabeled non-hierarchical one.

On one hand, we recall from 4.45 that, for all $x, y \in \widehat{\Lambda}_{N},\left(X^{N, x}, Y^{N, y}\right)$ denotes the Markov process on $\widehat{\Lambda}_{N} \times \widehat{\Lambda}_{N}$ started from $(x, y)$ and with generator $B^{N}$ defined in (2.14). We refer to such process as the non-hierarchical or symmetric process and recall that $\mathrm{P}^{N}$ and $\mathrm{E}^{N}$ denote their probability law and corresponding expectation, respectively. On the other hand, we define by $\left(\widetilde{X}^{N, x}, \widetilde{Y}^{N, y}\right)$ the so-called hierarchical or lookdown Markov process on $\widehat{\Lambda}_{N} \times \widehat{\Lambda}_{N}$ started from $(x, y)$ and with generator $C^{N}$ given, for all functions $f: \widehat{\Lambda}_{N} \times \widehat{\Lambda}_{N} \rightarrow \mathbb{R}$, by

$$
\begin{aligned}
C^{N} f(x, y) & :=A^{N} f(\cdot, y)(x)+A^{N} f(x, \cdot)(y) \\
& +\mathbf{1}_{\left\{x, y \in \Lambda_{N}\right\}} \mathbf{1}_{\{|x-y|=1\}} 2(f(x, x)-f(x, y)) .
\end{aligned}
$$

We let $\widetilde{\mathrm{P}}^{N}$ and $\widetilde{\mathrm{E}}^{N}$ denote the probability law and corresponding expectation, respectively. We emphasize that the hierarchical dynamics described by the generator $C^{N}$ in 4.50 dictates that the interaction part of the dynamics (the second line in the r.h.s. in (4.50) affects only the second class particle and compensates this asymmetry by doubling the rate of the interaction. 
Proposition 4.1. For all $\beta \in[0, \infty), N \in \mathbb{N},(x, y) \in \widehat{\Lambda}_{N} \times \widehat{\Lambda}_{N}$ and $t \geq 0$, we have, for all symmetric functions $f: \widehat{\Lambda}_{N} \times \widehat{\Lambda}_{N} \rightarrow \mathbb{R}$,

$$
\mathrm{E}^{N}\left[f\left(X_{t}^{N, x}, Y_{t}^{N, y}\right)\right]=\widetilde{\mathrm{E}}^{N}\left[f\left(\widetilde{X}_{t}^{N, U}, \widetilde{Y}_{t}^{N, V}\right)\right],
$$

where the random variables $(U, V)$ take the values $(x, y) \in \widehat{\Lambda}_{N} \times \widehat{\Lambda}_{N}$ or $(y, x) \in \widehat{\Lambda}_{N} \times \widehat{\Lambda}_{N}$ with equal probability.

Proof. As mentioned above, this result is a particular case of the more general lookdown construction for the multi-type Moran model with mutation (see, e.g., [15]). However, for the convenience of the reader, we report the short proof below. Indeed, it suffices to show that, for all symmetric functions $f: \widehat{\Lambda}_{N} \times \widehat{\Lambda}_{N} \rightarrow \mathbb{R}$ and for all $(x, y) \in \widehat{\Lambda}_{N} \times \widehat{\Lambda}_{N}$, we have

$$
B^{N} f(x, y)=\frac{1}{2}\left(C^{N} f(x, y)+C^{N} f(y, x)\right),
$$

where we recall that the operator $C^{N}$ was defined in 4.50 . This is indeed the case:

$$
\begin{aligned}
B^{N} f(x, y) & =A^{N} f(\cdot, y)(x)+A^{N} f(x, \cdot)(y)+\mathbf{1}_{\left\{x, y \in \Lambda_{N}\right\}} \mathbf{1}_{\{|x-y|=1\}}(f(x, x)+f(y, y)-2 f(x, y)) \\
& =\frac{1}{2}\left(A^{N} f(\cdot, y)(x)+A^{N} f(y, \cdot)(x)+A^{N} f(x, \cdot)(y)+A^{N} f(\cdot, x)(y)\right) \\
& +\mathbf{1}_{\left\{x, y \in \Lambda_{N}\right\}} \mathbf{1}_{\{|x-y|=1\}}((f(x, x)-f(x, y))+(f(y, y)-f(y, x))) \\
& =\frac{1}{2}\left(C^{N} f(x, y)+C^{N} f(y, x)\right) .
\end{aligned}
$$

Because $B^{N}$ maps symmetric functions into symmetric functions, by induction, a similar identity holds for all $\ell \in \mathbb{N}_{0}$ and $x, y \in \widehat{\Lambda}_{N}$,

$$
\left(B^{N}\right)^{\ell} f(x, y)=\frac{1}{2}\left(\left(C^{N}\right)^{\ell} f(x, y)+\left(C^{N}\right)^{\ell} f(y, x)\right),
$$

yielding, for all $t \geq 0$,

$$
e^{t B^{N}} f(x, y)=\frac{1}{2}\left(e^{t C^{N}} f(x, y)+e^{t C^{N}} f(y, x)\right), \quad(x, y) \in \widehat{\Lambda}_{N} \times \widehat{\Lambda}_{N} .
$$

Remark 4.2 ( $n$-CLASS LOOKDOWN INCLUSION PARTICLE SYSTEMS). One may introduce an analogous hierarchical "lookdown" construction with more than two, say $n>2$, inclusion particles, in which the $k$-th class particle $(k \leq n)$ evolves not being affected by the particles of class $\ell>k$ and joins at rate 2 any neighboring particle in the bulk of class $\ell<k$. Along the same lines, if the class labels are uniformly randomized at the initial time, then, at any later time, the probability law of the unlabeled hierarchical coincide with that of the unlabeled non-hierarchical inclusion process started from the same initial configuration. However, for our purposes, we only need this equivalence for systems with two particles.

Proof of Lemma 4.3. In view of Proposition 4.1 and because the function $g_{N}: \widehat{\Lambda}_{N} \times \widehat{\Lambda}_{N} \rightarrow \mathbb{R}$ defined in (4.47) is symmetric, i.e., $g_{N}(x, y)=g_{N}(y, x)$ for all $(x, y) \in \widehat{\Lambda}_{N} \times \widehat{\Lambda}_{N}$, we have

$$
\begin{aligned}
\sup _{x, y \in \Lambda_{N}} & \int_{0}^{\infty} S_{s}^{N} g_{N}(x, y) \mathrm{d} s \\
= & \sup _{x, y \in \Lambda_{N}} \int_{0}^{\infty} \mathrm{E}^{N}\left[g_{N}\left(X_{s}^{N, x}, Y_{s}^{N, y}\right)\right] \mathrm{d} s \\
& =\sup _{x, y \in \Lambda_{N}} \int_{0}^{\infty} \frac{1}{2}\left(\widetilde{\mathrm{E}}^{N}\left[g_{N}\left(\widetilde{X}_{s}^{N, x}, \widetilde{Y}_{s}^{N, y}\right)\right]+\widetilde{\mathrm{E}}^{N}\left[g_{N}\left(\widetilde{X}_{s}^{N, y}, \widetilde{Y}_{s}^{N, x}\right)\right]\right) \mathrm{d} s .
\end{aligned}
$$

Moreover, by conditioning on the non-absorption of the first class inclusion particle, we further obtain, for all $x, y \in \widehat{\Lambda}_{N}$,

$$
\widetilde{\mathrm{E}}^{N}\left[g_{N}\left(\widetilde{X}_{s}^{N, x}, \widetilde{Y}_{s}^{N, y}\right)\right]=\widetilde{\mathrm{P}}^{N}\left(\left(\widetilde{X}_{s}^{N, x}, \widetilde{Y}_{s}^{N, y}\right) \in \Lambda_{N} \times \Lambda_{N}\right)
$$




$$
\begin{aligned}
& =\widetilde{\mathrm{P}}^{N}\left(\widetilde{Y}_{s}^{N, y} \in \Lambda_{N} \mid \widetilde{X}_{s}^{N, x} \in \Lambda_{N}\right) \widetilde{\mathrm{P}}^{N}\left(\widetilde{X}_{s}^{N, x} \in \Lambda_{N}\right) \\
& \leq \widetilde{\mathrm{P}}^{N}\left(\widetilde{X}_{s}^{N, x} \in \Lambda_{N}\right)
\end{aligned}
$$

which yields

$$
\begin{aligned}
\sup _{x, y \in \Lambda_{N}} \int_{0}^{\infty} S_{s}^{N} g_{N}(x, y) \mathrm{d} s & \leq \sup _{x \in \Lambda_{N}} \int_{0}^{\infty} \widetilde{\mathrm{P}}^{N}\left(\widetilde{X}_{s}^{N, x} \in \Lambda_{N}\right) \mathrm{d} s \\
& =\sup _{x \in \Lambda_{N}} \int_{0}^{\infty} \mathrm{P}^{N}\left(X_{s}^{N, x} \in \Lambda_{N}\right) \mathrm{d} s,
\end{aligned}
$$

where we recall that the law of the first class particle $\widetilde{X}^{N, x}$ coincides, by definition, with that of the random walk $X^{N, x}$ on $\widehat{\Lambda}_{N}$ with generator $A^{N}$. By Lemma B.1 below, the r.h.s. in 4.51) is bounded above by

$$
C \max \left\{1, N^{\beta-1}\right\}
$$

for some constant $C>0$ independent of $N \in \mathbb{N}$, yielding, in conclusion, 4.49).

\section{Appendix A. Construction of test function spaces}

In this section, we construct the function spaces $\mathscr{S}$ whose elements serve as test functions for the $\mathscr{S}^{\prime}$-valued empirical density fields. The setting resembles that in, e.g., 25, 5, although we consider a different family of Hilbertian seminorms which turn $\mathscr{S}$ into a nuclear Fréchet space.

We start by recalling some definitions and facts (35, Chapter 11] and [34, Chapter 1]). Let $L^{2}([0,1])$ be endowed with the standard scalar product $\langle\cdot, \cdot\rangle, \mathcal{C}^{\infty}([0,1])$ be the linear subspace of elements of $L^{2}([0,1])$ with a smooth representative function on $(0,1)$, whose derivatives are uniformly continuous and, thus, may be continuously extended on $[0,1]$ and $\mathcal{C}_{\mathrm{c}}^{\infty}([0,1])$ the subspace of $\mathcal{C}^{\infty}([0,1])$ of compactly supported functions on $(0,1)$. Then

$$
\mathcal{C}_{\mathrm{c}}^{\infty}([0,1]) \subset \mathcal{C}^{\infty}([0,1]) \subset L^{2}([0,1])
$$

with $\mathcal{C}_{\mathrm{c}}^{\infty}([0,1])$ and, thus, $\mathcal{C}^{\infty}([0,1])$ being dense subspaces of $L^{2}([0,1])$. The general framework will be the following: for all $\beta \geq 0$, we consider a densely defined, closed and self-adjoint operator $\mathcal{L}$ with domain $D(\mathcal{L})$ and such that $\langle F, \mathcal{L} F\rangle \geq 0$ for all $F \in D(\mathcal{L})$. Such a self-adjoint operator will arise as associated with a suitable bilinear form $(\mathcal{E}, D(\mathcal{E}))$. Moreover, $\left\{\mathcal{T}_{t}: t \geq 0\right\}$ will denote the semigroup on $L^{2}([0,1])$ associated with $\mathcal{A}:=-\mathcal{L}$. Then we verify the following property (see [34, Eq. (1.3.17)]):

$$
\exists k_{*} \in \mathbb{N} \text { such that }(I+\mathcal{L})^{-\frac{k_{*}}{2}} \quad \text { is Hilbert-Schmidt . }
$$

By following the construction in [34, Example 1.3.2], we get that there exist $\left\{\lambda_{n}: n \in \mathbb{N}_{0}\right\} \subset[0, \infty)$ with $0 \leq \lambda_{0} \leq \lambda_{1} \leq \ldots$ and an orthonormal basis $\left\{\psi_{n}: n \in \mathbb{N}_{0}\right\}$ in $L^{2}([0,1])$ such that

$$
\mathcal{L} \psi_{n}=\lambda_{n} \psi_{n}, \quad \forall n \in \mathbb{N}_{0} .
$$

Moreover, we define the space

$$
\begin{aligned}
\mathscr{S} & :=\bigcap_{k \in \mathbb{Z}}\left\{F \in L^{2}([0,1]):\left\|(I+\mathcal{L})^{\frac{k}{2}} F\right\|_{L^{2}([0,1])}^{2}<\infty\right\} \\
& =\bigcap_{k \in \mathbb{Z}}\left\{F \in L^{2}([0,1]): \sum_{n \in \mathbb{N}_{0}}\left(1+\lambda_{n}\right)^{k}\left(\left\langle F, \psi_{n}\right\rangle\right)^{2}<\infty\right\}
\end{aligned}
$$

the inner products on $\mathscr{S}$ given, for all $k \in \mathbb{Z}$, by

$$
\langle F, G\rangle_{k}:=\sum_{n \in \mathbb{N}_{0}}\left(1+\lambda_{n}\right)^{k}\left\langle F, \psi_{n}\right\rangle\left\langle G, \psi_{n}\right\rangle
$$


and, for all $k \in \mathbb{Z}, \mathcal{H}_{k}$ as the completion of $\mathscr{S}$ with respect to $\langle\cdot, \cdot\rangle_{k}$. Note that, by the assumed density of $D(\mathcal{L}), \mathcal{H}_{0}=L^{2}([0,1])$. Moreover, for all $k \in \mathbb{Z}$, by Friedrichs extension, we have

$$
\mathcal{H}_{k}=D\left((I+\mathcal{L})^{\frac{k}{2}}\right) .
$$

As a consequence of these definitions,

$$
\langle F, F\rangle_{k} \geq\langle F, F\rangle_{\ell}, \quad \text { for all } \quad k \geq \ell,
$$

and, by A.1 , all the canonical embeddings $\mathcal{H}_{k} \hookrightarrow \mathcal{H}_{\ell}$ with $k \geq \ell+k_{*}$ are Hilbert-Schmidt. This will ensure that $\mathscr{S}$ endowed with the locally convex topology induced by the family of increasing Hilbertian norms

$$
\left\{\|\cdot\|_{k}:=\sqrt{\langle\cdot, \cdot\rangle_{k}}: k \in \mathbb{Z}\right\}
$$

is a nuclear Fréchet space with topological dual space $\mathscr{S}^{\prime}$ given by

$$
\mathscr{S}^{\prime}=\bigcup_{k \in \mathbb{Z}} \mathcal{H}_{k} .
$$

Moreover, the semigroup $\left\{\mathcal{T}_{t}: t \geq 0\right\}$ on $L^{2}([0,1])$ determined by $\mathcal{A}$ is a strongly continuous contraction semigroup described by

$$
\mathcal{T}_{t} F=\sum_{n \in \mathbb{N}_{0}} e^{-\lambda_{n} t}\left\langle F, \psi_{n}\right\rangle \psi_{n}, \quad F \in L^{2}([0,1]),
$$

and is "compatible with $\left(\mathscr{S}, L^{2}([0,1]), \mathscr{S}^{\prime}\right)$ " [34, Definition 1.3.5] in the following sense:

(I) For all $t \geq 0, \mathcal{T}_{t} \mathscr{S} \subseteq \mathscr{S}$.

(II) The restriction $\left.\mathcal{T}_{t}\right|_{\mathscr{S}}: \mathscr{S} \rightarrow \mathscr{S}$ is continuous for all $t \geq 0$.

(III) For all $F \in \mathscr{S}, t \mapsto \mathcal{T}_{t} F$ is continuous.

(IV) $\left.\mathcal{A}\right|_{\mathscr{S}}: \mathscr{S} \rightarrow \mathscr{S}$ is continuous.

Given the above common framework, we list below the specific choices of self-adjoint operators $\mathcal{L}$ and associated forms $(\mathcal{E}, D(\mathcal{E}))$ for each of the three regimes of the parameter $\beta \geq 0$. In what follows, we let $\mathcal{W}^{k, p}$ with $k \in \mathbb{N}, p \geq 1$ denote the standard Sobolev spaces on $(0,1)$ (see, e.g., [1]).

Dirichlet $(\beta<1)$. For Dirichlet boundary conditions, we consider $\mathcal{L}$ as the unique self-adjoint operator associated with

$$
D(\mathcal{E})=\mathcal{W}_{0}^{1,2}:={\overline{\mathcal{C}_{\mathrm{c}}([0,1])}}^{\mathcal{W}^{1,2}}
$$

and

$$
\mathcal{E}(F, G):=\alpha \int_{[0,1]} \frac{\mathrm{d}}{\mathrm{d} u} F(u) \frac{\mathrm{d}}{\mathrm{d} u} G(u) \mathrm{d} u .
$$

Moreover,

$$
D(\mathcal{L})=\left\{F \in \mathcal{W}_{0}^{1,2}: \frac{\mathrm{d}^{2}}{\mathrm{~d} u^{2}} F \in L^{2}([0,1])\right\}
$$

(see, e.g., [3, Example 3.1] and references therein).

Robin $(\beta=1)$. For Robin boundary conditions, we consider $\mathcal{L}$ as the unique self-adjoint operator associated with

and

$$
D(\mathcal{E})=\mathcal{W}^{1,2}:=\left\{F \in L^{2}([0,1]): \frac{\mathrm{d}}{\mathrm{d} u} F \in L^{2}([0,1])\right\}
$$

$$
\mathcal{E}(F, G):=\alpha \int_{[0,1]} \frac{\mathrm{d}}{\mathrm{d} u} F(u) \frac{\mathrm{d}}{\mathrm{d} u} G(u) \mathrm{d} u+\alpha_{L} F(0) G(0)-\alpha_{R} F(1) G(1) .
$$

Moreover,

$$
D(\mathcal{L})=\left\{F \in L^{2}([0,1]): \frac{\mathrm{d}^{2}}{\mathrm{~d} u^{2}} F \in L^{2}([0,1]), \frac{\mathrm{d}^{+}}{\mathrm{d} u} F(0)=\frac{\alpha_{L}}{\alpha} F(0), \frac{\mathrm{d}^{-}}{\mathrm{d} u} F(1)=\frac{\alpha_{R}}{\alpha} F(1)\right\}
$$


(see, e.g., [3]).

Neumann $(\beta>1)$. For Neumann boundary conditions, we consider $\mathcal{L}$ as the unique self-adjoint operator associated with

$$
D(\mathcal{E})=\mathcal{W}^{1,2}
$$

and

$$
\mathcal{E}(F, G):=\alpha \int_{[0,1]} \frac{\mathrm{d}}{\mathrm{d} u} F(u) \frac{\mathrm{d}}{\mathrm{d} u} G(u) \mathrm{d} u
$$

Moreover,

$$
D(\mathcal{L})=\left\{F \in \mathcal{W}^{1,2}: \frac{\mathrm{d}^{2}}{\mathrm{~d} u^{2}} F \in L^{2}([0,1]) \text { and } \text { A.5 below holds }\right\}
$$

where

$$
\int_{[0,1]} \frac{\mathrm{d}^{2}}{\mathrm{~d} u^{2}} F(u) G(u) \mathrm{d} u=-\int_{[0,1]} \frac{\mathrm{d}}{\mathrm{d} u} F(u) \frac{\mathrm{d}}{\mathrm{d} u} G(u) d u, \quad \text { for all } G \in \mathcal{W}^{1,2} .
$$

(See, e.g., [3, Example 3.2]).

From classical results on the eigenvalues of the Dirichlet, Robin and Neumann Laplacian operators on the interval $[0,1]$ (see, e.g., 42]), we know that, for all $\beta \geq 0$, the self-adjoint operator $\mathcal{L}$ has a discrete non-negative spectrum. Moreover, by the ordering of Neumann, Robin and Dirichlet eigenvalues (see, e.g., [2]) and by Weyl's law (see, e.g., [42]), if we let, for all $\beta \geq 0$,

$$
\left\{\lambda_{n}: n \in \mathbb{N}_{0}\right\} \subset[0, \infty)
$$

denote the eigenvalues associated to the self-adjoint operator $\mathcal{L}$, there exists a constant $\Lambda=\Lambda_{\beta} \in$ $(0, \infty)$ for which we have:

$$
\frac{\sqrt{\lambda_{n}}}{n} \underset{n \rightarrow \infty}{\longrightarrow} \Lambda
$$

As a consequence of A.6, we get property A.1 with $k_{*}=1$. This property enables the construction of the nuclear Fréchet spaces $\mathscr{S}$ and their topological duals $\mathscr{S}^{\prime}$ as above.

Let us further characterize such spaces by proving Proposition 2.2 .

Proof of Proposition 2.2. Let us first prove that $\mathscr{S}$ consists of smooth functions with uniformly continuous derivatives of any order. By A.3, we have

$$
\mathscr{S}=\bigcap_{k \in \mathbb{Z}} D\left((I+\mathcal{L})^{\frac{k}{2}}\right) \subseteq \mathcal{C}^{\infty}([0,1]) .
$$

Indeed, the last inclusion is a consequence of

$$
D\left((I+\mathcal{L})^{\frac{k}{2}}\right) \subseteq \mathcal{W}^{k, 2}, \quad k \in \mathbb{N}_{0},
$$

and the Sobolev embedding theorems (see, e.g., [1, Theorem 5.4.II.C']): for all $k \in \mathbb{N}_{0}$ and $\lambda \in\left(0, \frac{1}{2}\right]$,

$$
\mathcal{W}^{k+1,2} \subseteq \mathcal{C}^{k, \lambda}([0,1]),
$$

where $\mathcal{C}^{k, \lambda}([0,1])$ denotes the subspace of $\mathcal{C}^{k}([0,1])$ whose derivatives up to order $k$ are Hölder continuous with Hölder exponent $\lambda$ (see, e.g., [1, §1.27]). Moreover, the embedding (A.7) into the Banach space $\left(\mathcal{C}^{k, \lambda}([0,1]),\|\cdot\|_{k, \lambda}\right)$ is continuous.

Next, let us show which boundary conditions the test functions satisfy. We observe that, for all $\beta \geq 0$, if $G \in \mathscr{S}$, then

$$
G=\sum_{n \in \mathbb{N}_{0}}\left\langle G, \psi_{n}\right\rangle \psi_{n}
$$


where $\left\{\psi_{n}: n \in \mathbb{N}_{0}\right\}$ denotes the orthonormal basis in $L^{2}([0,1])$ of eigenfunctions of $\mathcal{L}$. Moreover, by A.2,

$$
\left\{\psi_{n}: n \in \mathbb{N}_{0}\right\} \subseteq \bigcap_{k \in \mathbb{N}_{0}} D\left(\mathcal{L}^{k}\right) \subseteq \mathscr{S}
$$

In particular, because of the definitions of $\mathcal{L}$ and their domains $D(\mathcal{L})$, the eigenfunctions $\left\{\psi_{n}: n \in \mathbb{N}_{0}\right\}$ satisfy the corresponding boundary conditions (2.17)-2.19). Therefore, if we show that, for all $\beta \geq 0, G \in \mathscr{S}$ and $\ell \in \mathbb{N}_{0}$,

$$
\sum_{n \in \mathbb{N}_{0}}\left|\left\langle G, \psi_{n}\right\rangle\right| \sup _{u \in[0,1]}\left|\left(\frac{\mathrm{d}}{\mathrm{d} u}\right)^{\ell} \psi_{n}(u)\right|<\infty,
$$

then, by A.8, we get

$$
\left(\frac{\mathrm{d}}{\mathrm{d} u}\right)^{\ell} G=\sum_{n \in \mathbb{N}_{0}}\left\langle G, \psi_{n}\right\rangle\left(\frac{\mathrm{d}}{\mathrm{d} u}\right)^{\ell} \psi_{n},
$$

and the conclusion follows. To this purpose, let us prove that

$$
\sup _{u \in[0,1]}\left(\left(\frac{\mathrm{d}}{\mathrm{d} u}\right)^{\ell} \psi_{n}(u)\right)^{2} \leq \sum_{h=0}^{\ell}\left(\alpha_{L}+\alpha_{R}\right)^{2 h} \lambda_{n}^{\ell+1-h}
$$

holds true for all $\beta \geq 0, n \in \mathbb{N}_{0}$ and $\ell \in \mathbb{N}_{0}$. Indeed, Cauchy-Schwarz inequality and the boundary conditions satisfied by the eigenfunction $\psi_{n}$ yield

$$
\begin{aligned}
\sup _{u \in[0,1]} & \left(\left(\frac{\mathrm{d}}{\mathrm{d} u}\right)^{\ell} \psi_{n}(u)\right)^{2} \\
& \leq \int_{[0,1]}\left(\left(\frac{\mathrm{d}}{\mathrm{d} u}\right)^{\ell+1} \psi_{n}(u)\right)^{2} d u \\
& =\lambda_{n}^{\ell+1}+\mathbf{1}_{\{\beta=1\}}\left\{-\alpha_{L}\left(\left.\left(\frac{\mathrm{d}}{\mathrm{d} u}\right)^{\ell}\right|_{u=0} \psi_{n}\right)^{2}+\alpha_{R}\left(\left.\left(\frac{\mathrm{d}}{\mathrm{d} u}\right)^{\ell}\right|_{u=1} \psi_{n}\right)^{2}\right\} \\
& \leq \lambda_{n}^{\ell+1}+\mathbf{1}_{\{\beta=1\}}\left(\alpha_{L}+\alpha_{R}\right)^{2}\left\{\sup _{u \in[0,1]}\left(\left(\frac{\mathrm{d}}{\mathrm{d} u}\right)^{\ell-1} \psi_{n}(u)\right)^{2}\right\},
\end{aligned}
$$

and, by iterating, we get A.10. As a consequence of A.10 and Cauchy-Schwarz inequality, we get, for all $\left\{a_{n}: n \in \mathbb{N}\right\} \subset(0, \infty)$,

$$
\begin{aligned}
& \sum_{n \in \mathbb{N}_{0}}\left|\left\langle G, \psi_{n}\right\rangle\right| \sup _{u \in[0,1]}\left|\left(\frac{\mathrm{d}}{\mathrm{d} u}\right)^{\ell} \psi_{n}(u)\right| \\
& \leq \sqrt{\sum_{n \in \mathbb{N}_{0}}\left(\left\langle G, \psi_{n}\right\rangle\right)^{2} a_{n}^{2} \sup _{u \in[0,1]}\left(\left(\frac{\mathrm{d}}{\mathrm{d} u}\right)^{\ell} \psi_{n}(u)\right)^{2}} \sqrt{\sum_{n \in \mathbb{N}_{0}} a_{n}^{-2}} \\
& \leq \sqrt{\sum_{h=0}^{\ell}\left(\alpha_{L}+\alpha_{R}\right)^{2 h} \sum_{n \in \mathbb{N}_{0}}\left(\left\langle G, \psi_{n}\right\rangle\right)^{2} a_{n}^{2} \lambda_{n}^{\ell+1-h}} \sqrt{\sum_{n \in \mathbb{N}_{0}} a_{n}^{-2}} .
\end{aligned}
$$

By choosing $\left\{a_{n}: n \in \mathbb{N}_{0}\right\}=\left\{\lambda_{n}: n \in \mathbb{N}_{0}\right\}$, by definition of $G \in \mathscr{S}$ (cf. A.3) and A.6, we obtain the uniform convergence of the series on the r.h.s. of A.9). This concludes the proof. 
A.1. A remark on the topologies of $\mathcal{C}\left([0, \infty), \mathscr{S}^{\prime}\right)$ and $\mathcal{D}\left([0, \infty), \mathscr{S}^{\prime}\right)$. We remark that we have defined, for all $\beta \geq 0, \mathscr{S}^{\prime}$ as the dual of $\mathscr{S}$ endowed with the strong topology. However, when considering the spaces $\mathcal{C}\left([0, \infty), \mathscr{S}^{\prime}\right)$ and $\mathcal{D}\left([0, \infty), \mathscr{S}^{\prime}\right)$, by [44, Lemma 3.2], the strong topology may be replaced by the weak* topology (see below) when considering Borel probability measures on such spaces because the Borel $\sigma$-fields induced by weak* and strong topologies coincide. More precisely, let us recall from [34 that:

(1) For all $G \in \mathscr{S},\|\cdot\|_{G}: \mathscr{S}^{\prime} \rightarrow[0, \infty)$ given, for all $f \in \mathscr{S}^{\prime}$, by

$$
\|f\|_{G}:=|\langle G, f\rangle| \text {, }
$$

is a seminorm. The family $\left\{\|\cdot\|_{G}: G \in \mathscr{S}\right\}$ determines the weak* topology on $\mathscr{S}^{\prime}$ (see [34, Definition 1.1.3]) and, in particular, $\mathscr{U} \subset \mathscr{S}^{\prime}$ is a weak* neighborhood of $f \in \mathscr{S}^{\prime}$ if there exist $n \in \mathbb{N},\left\{G_{1}, \ldots, G_{n}\right\} \subset \mathscr{S}$ and $\left\{\varepsilon_{1}, \ldots, \varepsilon_{n}\right\} \subset(0, \infty)$ such that

$$
\mathscr{U}=\left\{g \in \mathscr{S}^{\prime}:\|f-g\|_{G_{k}}<\varepsilon_{k} \text { for all } k=1, \ldots, n\right\} .
$$

(2) For all $G \in \mathscr{S}$ and $T>0$,

$$
\|f\|_{G, T}:=\sup _{t \in[0, T]}\|f(t)\|_{G}
$$

with $f=\{f(t): t \geq 0\} \in \mathcal{C}\left([0, \infty), \mathscr{S}^{\prime}\right)$ defines a seminorm on $\mathcal{C}\left([0, \infty), \mathscr{S}^{\prime}\right)$. The family $\left\{\|\cdot\|_{G, T}: G \in \mathscr{S}, T>0\right\}$ defines the weak* topology of $\mathcal{C}\left([0, \infty), \mathscr{S}^{\prime}\right)$ (see [34, p. 73]), with neighborhoods $\mathscr{U} \subset \mathscr{S}^{\prime}$ of $f=\{f(t): t \geq 0\} \in \mathcal{C}\left([0, \infty), \mathscr{S}^{\prime}\right)$ given by finite intersections of sets of the following type:

$$
\left\{g \in \mathcal{C}\left([0, \infty), \mathscr{S}^{\prime}\right):\|f-g\|_{G, T}<\varepsilon\right\} .
$$

(3) The weak* topology of $\mathcal{D}\left([0, \infty), \mathscr{S}^{\prime}\right)$ is defined in terms of the following pseudometrics 34 , p. 71]: for all $G \in \mathscr{S}, T>0$ and $f, g \in \mathcal{D}\left([0, \infty), \mathscr{S}^{\prime}\right)$,

$$
d_{G, T}(f, g):=\inf _{\lambda \in \Lambda_{T}}\left\{\sup _{t \in[0, T]}\|f(t)-g(\lambda(t))\|_{G}+\gamma(\lambda)\right\},
$$

where $\Lambda_{T}$ is the set of strictly increasing continuous maps from $[0, T]$ onto itself and such that

$$
\gamma(\lambda):=\sup _{s, t \in[0, T]}\left|\log \left(\frac{\lambda(t)-\lambda(s)}{t-s}\right)\right|<\infty .
$$

Neighborhoods of $f \in \mathcal{D}\left([0, \infty), \mathscr{S}^{\prime}\right)$ consist of finite intersections of sets of the following type:

$$
\left\{g \in \mathcal{D}\left([0, \infty), \mathscr{S}^{\prime}\right): d_{G, T}(f, g)<\varepsilon\right\} .
$$

As a consequence of the above definitions and [44, Lemma 3.2], a sequence $\left\{\mathscr{P}^{N}: N \in \mathbb{N}\right\}$ of Borel probability measures in $\mathcal{D}\left([0, \infty), \mathscr{S}^{\prime}\right)$ converges in probability to $f \in \mathcal{D}\left([0, \infty), \mathscr{S}^{\prime}\right)$ if, for all $T>0, G \in \mathscr{S}$ and $\delta>0$,

$$
\mathscr{P}^{N}\left(\left\{g \in \mathcal{D}\left([0, \infty), \mathscr{S}^{\prime}\right): d_{G, T}(f, g)>\delta\right\}\right) \underset{N \rightarrow \infty}{\longrightarrow} 0 .
$$

If, in particular, $f \in \mathcal{C}\left([0, \infty), \mathscr{S}^{\prime}\right)$ and if

$$
\mathscr{P}^{N}\left(\left\{g \in \mathcal{D}\left([0, \infty), \mathscr{S}^{\prime}\right):\|f-g\|_{G, T}>\delta\right\}\right) \underset{N \rightarrow \infty}{\longrightarrow} 0
$$

holds for all $T>0, G \in \mathscr{S}$ and $\delta>0$, A.11 follows. This notion of convergence in probability to a Dirac measure turns out to be equivalent to weak convergence in $\mathcal{D}\left([0, \infty), \mathscr{S}^{\prime}\right)$. Indeed, this follows from a version of Portmanteau's theorem in the context of completely regular Hausdorff topological spaces and limiting $\tau$-additive measures (see, e.g., 9, Corollary II.8.2.4]). Combined with the above 
considerations, a sequence $\left\{\mathscr{P}^{N}: N \in \mathbb{N}\right\}$ of Borel probability measures on $\mathcal{D}\left([0, \infty), \mathscr{S}^{\prime}\right)$ converges (either weakly or in probability) to the Dirac measure supported on $f \in \mathcal{C}\left([0, \infty), \mathscr{S}^{\prime}\right)$ if and only if

$$
\mathscr{P}^{N}\left(\left\{g \in \mathcal{D}\left([0, \infty), \mathscr{S}^{\prime}\right): \sup _{t \in[0, T]}|\langle G, g(t)\rangle-\langle G, f(t)\rangle|>\delta\right\}\right) \underset{N \rightarrow \infty}{\longrightarrow} 0
$$

holds for all $T>0, G \in \mathscr{S}$ and $\delta>0$.

\section{Appendix B. Absorbing Random WalK's estimate}

In order to study absorption probabilities before a given time for the dual random walk

$$
\left\{X_{t}^{N, x}: t \geq 0\right\}
$$

on $\widehat{\Lambda}_{N}$ with generator $A^{N}$ given in 2.10$)$, we employ Stone's pathwise construction of birth-anddeath processes from a time-change of Brownian motion paths (see [46]). To this purpose, $\mathrm{P}$ and $\mathrm{E}$ denote the probability law and corresponding expectation of the underlying one-dimensional standard Brownian motion $\left\{B_{t}: t \geq 0\right\}$ with $B_{0}=0$ a.s. and $\mathrm{E}\left[\left(B_{t}\right)^{2}\right]=t$. Let us briefly describe such construction and introduce some notation.

We first define a (singular with respect to Lebesgue) measure $\nu^{N}$ on $\mathbb{R}$ which yields the correct time-change of Brownian motion paths. More precisely, $\nu^{N}$ has the following form

$$
\nu^{N}:=\sum_{x \in \mathbb{Z}} w_{x}^{N} \delta_{z_{x}^{N}}
$$

where $\left\{w_{x}^{N}: x \in \mathbb{Z}\right\}$ are called the "weights" and are given by

$$
w_{x}^{N}=\frac{\alpha}{2 N}, \quad x \in \mathbb{Z},
$$

while $\left\{z_{x}^{N}: x \in \mathbb{Z}\right\} \subset \mathbb{R}$, satisfying the order relation $z_{x}^{N}<z_{x+1}^{N}$ for all $x \in \mathbb{Z}$ and given, for $x \in \widehat{\Lambda}_{N}$, by

$$
\begin{aligned}
z_{0}^{N} & =0 \\
z_{1}^{N} & =\frac{1}{\alpha_{L} \alpha N^{1-\beta}} \\
& \vdots \\
z_{x}^{N} & =\frac{1}{\alpha_{L} \alpha N^{1-\beta}}+(x-1) \frac{1}{\alpha^{2} N} \\
& \vdots \\
z_{N}^{N} & =\frac{1}{\alpha_{L} \alpha N^{1-\beta}}+(N-2) \frac{1}{\alpha^{2} N}+\frac{1}{\alpha_{R} \alpha N^{1-\beta}},
\end{aligned}
$$

stands for the "support" of $\nu^{N}$. The specific choice of the support points $\left\{z_{x}^{N}: x \in \mathbb{Z} \backslash \widehat{\Lambda}_{N}\right\}$ is irrelevant for our purposes. Let us note that, by (B.1), there exists a constant $C>0$ such that, for all $\beta \in \mathbb{R}$,

$$
0<\inf _{N \in \mathbb{N}} \frac{z_{N}^{N}-z_{0}^{N}}{\max \left\{1, N^{\beta-1}\right\}} \leq \sup _{N \in \mathbb{N}} \frac{z_{N}^{N}-z_{0}^{N}}{\max \left\{1, N^{\beta-1}\right\}} \leq \frac{C}{2 \alpha}
$$

Let $\left\{\ell_{t}^{N, x}(z):(t, z) \in[0, \infty) \times \mathbb{R}\right\}$ denote the local time of $\left\{B_{t}+z_{x}^{N}: t \geq 0\right\}$ (see, e.g., [46, Theorem (Trotter)]). Hence,

$$
\psi_{t}^{N, x}=\int_{\mathbb{R}} \ell_{t}^{N, x}(z) \nu^{N}(\mathrm{~d} z)
$$


is the random $\nu^{N}$-weighted time that the Brownian motion has spent on the support of $\nu^{N}$ up to time $t \geq 0$. We note that $\psi^{N, x}:[0, \infty) \rightarrow[0, \infty)$ is a non-negative non-decreasing function. As a consequence of [46, §3], the process $\left\{Z_{t}^{N, x}: t \geq 0\right\}$ defined (a.s.) as

$$
Z_{t}^{N, x}:=B_{\phi_{t}^{N, x}}+z_{x}^{N}
$$

with $\left\{\phi_{t}^{N, x}: t \geq 0\right\}$ being the generalized inverse of $\left\{\psi_{t}^{N, x}: t \geq 0\right\}$, namely

$$
\phi_{t}^{N, x}=\sup \left\{s \geq 0: \psi_{s}^{N, x} \leq t\right\},
$$

is a jump process on $\left\{z_{y}^{N}, y \in \mathbb{Z}\right\}$ with nearest-neighbor jumps, starting from $z_{x}^{N} \in \mathbb{R}$, exit rates at $z_{y}^{N}$ given by

$$
\frac{1}{2 w_{y}^{N}} \frac{z_{y+1}^{N}-z_{y-1}^{N}}{\left(z_{y+1}^{N}-z_{y}^{N}\right)\left(z_{y}^{N}-z_{y-1}^{N}\right)}
$$

and jump probability from $z_{y}^{N}$ to $z_{y-1}^{N}$ given by

$$
\frac{z_{y+1}^{N}-z_{y}^{N}}{z_{y+1}^{N}-z_{y-1}^{N}} .
$$

In particular, the law of the process $\left\{Z_{t}^{N, x}: t \geq 0\right\}$ coincides with that of the process

$$
\left\{z_{X_{t}^{N, x}}^{N}: t \geq 0\right\}
$$

if we observe both processes until the first hitting of $\left\{z_{0}^{N}, z_{N}^{N}\right\}$. Ultimately, this construction stands at the core of the proof of Lemma B.1 because it allows us to write random walks' probabilities in terms of Brownian motion probabilities

$$
\mathrm{P}^{N}\left(X_{t}^{N, x} \in \Lambda_{N}\right)=\mathrm{P}\left(\psi_{\tau^{N, x}}^{N, x}>t\right),
$$

where $\tau^{N, x}$ denotes the first exit time from $\left(z_{0}^{N}, z_{N}^{N}\right) \subset \mathbb{R}$ of $\left\{B_{t}+z_{x}^{N}: t \geq 0\right\}$.

Lemma B.1. There exists a constant $C>0$ such that, for all $\beta \in \mathbb{R}$ and $N \in \mathbb{N}$, we have

$$
\sup _{x \in \Lambda_{N}} \int_{0}^{\infty} \mathrm{P}^{N}\left(X_{t}^{N, x} \in \Lambda_{N}\right) \mathrm{d} t \leq C \max \left\{1, N^{\beta-1}\right\} .
$$

Proof. In view of the identity in $\mathrm{B} .3$ and because $\psi_{\tau^{N, x}}^{N, x}$ is a non-negative random variable, we have, for all $\beta \in \mathbb{R}, N \in \mathbb{N}$ and $x \in \Lambda_{N}$,

$$
\int_{0}^{\infty} \mathrm{P}^{N}\left(X_{t}^{N, x} \in \Lambda_{N}\right) \mathrm{d} t=\int_{0}^{\infty} \mathrm{P}\left(\psi_{\tau^{N, x}}^{N, x}>t\right) \mathrm{d} t=\mathrm{E}\left[\psi_{\tau^{N, x}}^{N, x}\right] .
$$

By the definitions of $\nu^{N}, \psi^{N, x}$ and $\phi^{N, x}$ above, we have

$$
\psi_{\tau^{N, x}}^{N, x}=\frac{\alpha}{2 N} \sum_{y=1}^{N-1} \ell_{\tau^{N, x}}^{N, x}\left(z_{y}^{N}\right),
$$

and, thus,

$$
\mathrm{E}\left[\psi_{\tau^{N, x}}^{N, x}\right]=\frac{\alpha}{2 N} \sum_{y \in \Lambda_{N}} \mathrm{E}\left[\ell_{\tau^{N, x}}^{N, x}\left(z_{y}^{N}\right)\right] .
$$

Because the local times are non-negative random variables, we get, for all $N \in \mathbb{N}$ and $y \in \Lambda_{N}$,

$$
\begin{aligned}
\mathrm{E}\left[\ell_{\tau^{N, x}}^{N, x}\left(z_{y}^{N}\right)\right] & =\int_{0}^{\infty} \mathrm{P}\left(\ell_{\tau^{N, x}}^{N, x}\left(z_{y}^{N}\right)>t\right) \mathrm{d} t \\
& =\int_{0}^{\infty} \mathrm{P}\left(\ell_{\tau^{N, x}}^{N, x}\left(z_{y}^{N}\right)>t \text { and } B_{\tau^{N, x}}+z_{x}^{N}=z_{0}^{N}\right) \mathrm{d} t
\end{aligned}
$$




$$
+\int_{0}^{\infty} \mathrm{P}\left(\ell_{\tau^{N, x}}^{N, x}\left(z_{y}^{N}\right)>t \text { and } B_{\tau^{N, x}}+z_{x}^{N}=z_{N}^{N}\right) \mathrm{d} t .
$$

Let us provide, for all $N \in \mathbb{N}$, an upper bound uniform in $x$ and $y \in \Lambda_{N}$ for the first term on the r.h.s. above. To this purpose, we employ [10, Formula 3.3.6(a), p. 214]: for all $x, y \in \Lambda_{N}$,

$$
\mathrm{P}\left(\ell_{\tau^{N, x}}^{N, x}\left(z_{y}^{N}\right)>t \text { and } B_{\tau^{N, x}}+z_{x}^{N}=z_{0}^{N}\right)=C_{y}^{N, x} \exp \left(-D_{y}^{N} t\right),
$$

where

$$
C_{y}^{N, x}:= \begin{cases}\frac{z_{N}^{N}-z_{x}^{N}}{z_{N}^{N}-z_{0}^{N}} & \text { if } y<x \\ \frac{\left(z_{x}^{N}-z_{0}^{N}\right)\left(z_{N}^{N}-z_{y}^{N}\right)}{\left(z_{y}^{N}-z_{0}^{N}\right)\left(z_{N}^{N}-z_{0}^{N}\right)} & \text { if } y \geq x\end{cases}
$$

and

$$
D_{y}^{N}:=\frac{z_{N}^{N}-z_{0}^{N}}{2\left(z_{N}^{N}-z_{y}^{N}\right)\left(z_{y}^{N}-z_{0}^{N}\right)} .
$$

By integrating over time the expression in (B.7), we get

$$
\begin{aligned}
& \int_{0}^{\infty} \mathcal{P}\left(\ell_{\tau^{N, x}}^{N, x}\left(z_{y}^{N}\right)>t \text { and } B_{\tau^{N, x}}+z_{x}^{N}=z_{0}^{N}\right) \mathrm{d} t \\
& =2 \begin{cases}\frac{z_{N}^{N}-z_{x}^{N}}{\left(z_{N}^{N}-z_{0}^{N}\right)^{2}}\left(z_{N}^{N}-z_{y}^{N}\right)\left(z_{y}^{N}-z_{0}^{N}\right) & \text { if } y<x \\
\frac{z_{x}^{N}-z_{0}^{N}}{\left(z_{N}^{N}-z_{0}^{N}\right)^{2}}\left(z_{N}^{N}-z_{y}^{N}\right) & \text { if } y \geq x,\end{cases}
\end{aligned}
$$

from which we obtain the following upper bound, uniform in $x$ and $y \in \Lambda_{N}$ :

$$
\int_{0}^{\infty} \mathcal{P}\left(\ell_{\tau^{N, x}}^{N, x}\left(z_{y}^{N}\right)>t \text { and } B_{\tau^{N, x}}+z_{x}^{N}=z_{0}^{N}\right) \mathrm{d} t \leq 2\left(z_{N}^{N}-z_{0}^{N}\right) .
$$

An analogous argument yields

$$
\int_{0}^{\infty} \mathcal{P}\left(\ell_{\tau^{N, x}}^{N, x}\left(z_{y}^{N}\right)>t \text { and } B_{\tau^{N, x}}+z_{x}^{N}=z_{N}^{N}\right) \mathrm{d} t \leq 2\left(z_{N}^{N}-z_{0}^{N}\right),
$$

for all $x$ and $y \in \Lambda_{N}$. By (B.4), (B.5), (B.6), (B.8) and (B.9), we get:

$$
\int_{0}^{\infty} \mathrm{P}^{N}\left(X_{t}^{N, x} \in \Lambda_{N}\right) \mathrm{d} t \leq 2 \alpha\left(z_{N}^{N}-z_{0}^{N}\right)
$$

The upper bound in $(\mathrm{B} .2)$ concludes the proof.

\section{ACKNOWLEDGEMENTS}

F.S. wishes to thank Joe P. Chen for some fruitful discussions at an early stage of this work. C.F. and P.G. thank FCT/Portugal for support through the project UID/MAT/04459/2013. This project has received funding from the European Research Council (ERC) under the European Union's Horizon 2020 research and innovative programme (grant agreement No. 715734). F.S. thanks CAMGSD, IST, Lisbon, where part of this work has been done, and the European research and innovative programme No. 715734 for the kind hospitality. F.S. was founded by the European Union's Horizon 2020 research and innovation programme under the Marie-Skłodowska-Curie grant agreement No. 754411. 


\section{REFERENCES}

[1] R. A. Adams and J. J. F. Fournier. Sobolev spaces, volume 140 of Pure and Applied Mathematics (Amsterdam). Elsevier/Academic Press, Amsterdam, second edition, 2003.

[2] W. Arendt and M. Warma. Dirichlet and Neumann boundary conditions: What is in between? volume 3, pages 119-135. 2003. Dedicated to Philippe Bénilan.

[3] W. Arendt and M. Warma. The Laplacian with Robin boundary conditions on arbitrary domains. Potential Anal., 19(4):341-363, 2003.

[4] R. Baldasso, O. Menezes, A. Neumann, and R. R. Souza. Exclusion process with slow boundary. J. Stat. Phys., 167(5):1112-1142, 2017.

[5] C. Bernardin, P. Gonçalves, M. Jara, and S. Scotta. Equilibrium fluctuations for diffusive symmetric exclusion with long jumps and infinitely extended reservoirs. arXiv:2002.12841, 2020.

[6] C. Bernardin, P. Gonçalves, and B. Jiménez-Oviedo. A Microscopic Model for a One Parameter Class of Fractional Laplacians with Dirichlet Boundary Conditions. Arch. Ration. Mech. Anal., 239(1):1-48, 2021.

[7] A. Bianchi, S. Dommers, and C. Giardinà. Metastability in the reversible inclusion process. Electron. J. Probab., 22:Paper No. 70, 34, 2017.

[8] P. Billingsley. Convergence of probability measures. Wiley Series in Probability and Statistics: Probability and Statistics. John Wiley \& Sons, Inc., New York, second edition, 1999. A Wiley-Interscience Publication.

[9] V. I. Bogachev. Measure theory. Vol. I, II. Springer-Verlag, Berlin, 2007.

[10] A. N. Borodin and P. Salminen. Handbook of Brownian motion-facts and formulae. Probability and its Applications. Birkhäuser Verlag, Basel, second edition, 2002.

[11] G. Carinci, C. Franceschini, C. Giardinà, W. Groenevelt, and F. Redig. Orthogonal dualities of Markov processes and unitary symmetries. SIGMA Symmetry Integrability Geom. Methods Appl., 15:Paper No. 053, 27, 2019.

[12] G. Carinci, C. Giardinà, C. Giberti, and F. Redig. Duality for stochastic models of transport. J. Stat. Phys., 152(4):657-697, 2013.

[13] A. De Masi and E. Presutti. Mathematical Methods for Hydrodynamic Limits. Number 1501 in Lecture Notes in Mathematics. Springer-Verlag, 1991.

[14] B. Derrida, M. R. Evans, V. Hakim, and V. Pasquier. Exact solution of a 1D asymmetric exclusion model using a matrix formulation. J. Phys. A, 26(7):1493-1517, 1993.

[15] P. Donnelly and T. G. Kurtz. A countable representation of the Fleming-Viot measure-valued diffusion. Ann. Probab., 24(2):698-742, 1996.

[16] C. Erignoux. Hydrodynamic limit of boundary driven exclusion processes with nonreversible boundary dynamics. J. Stat. Phys., 172(5):1327-1357, 2018.

[17] C. Erignoux, P. Gonçalves, and G. Nahum. Hydrodynamics for SSEP with non-reversible slow boundary dynamics: Part I, the critical regime and beyond. J. Stat. Phys., 181(4):1433-1469, 2020.

[18] C. Erignoux, P. Gonçalves, and G. Nahum. Hydrodynamics for SSEP with non-reversible slow boundary dynamics: Part II, below the critical regime. ALEA Lat. Am. J. Probab. Math. Stat., 17(2):791-823, 2020.

[19] L. C. Evans. Partial differential equations, volume 19 of Graduate Studies in Mathematics. American Mathematical Society, Providence, RI, second edition, 2010.

[20] G. Eyink, J. L. Lebowitz, and H. Spohn. Hydrodynamics of stationary nonequilibrium states for some stochastic lattice gas models. Comm. Math. Phys., 132(1):253-283, 1990.

[21] G. Eyink, J. L. Lebowitz, and H. Spohn. Lattice gas models in contact with stochastic reservoirs: local equilibrium and relaxation to the steady state. Comm. Math. Phys., 140(1):119-131, 1991.

[22] J. Farfan, C. Landim, and M. Mourragui. Hydrostatics and dynamical large deviations of boundary driven gradient symmetric exclusion processes. Stochastic Process. Appl., 121(4):725-758, 2011.

[23] S. Floreani, F. Redig, and F. Sau. Orthogonal polynomial duality of boundary driven particle systems and non-equilibrium correlations. arXiv:2007.08272, 2020.

[24] T. Franco, P. Gonçalves, and A. Neumann. Phase transition in equilibrium fluctuations of symmetric slowed exclusion. Stochastic Process. Appl., 123(12):4156-4185, 2013.

[25] T. Franco, P. Gonçalves, and A. Neumann. Equilibrium fluctuations for the slow boundary exclusion process. In From particle systems to partial differential equations, volume 209 of Springer Proc. Math. Stat., pages 177-197. Springer, Cham, 2017.

[26] S. Frómeta, R. Misturini, and A. Neumann. The boundary driven zero-range process. arXiv:2006.13479 2020.

[27] C. Giardinà, J. Kurchan, and F. Redig. Duality and exact correlations for a model of heat conduction. J. Math. Phys., 48(3):033301, 15, 2007.

[28] C. Giardinà, J. Kurchan, F. Redig, and K. Vafayi. Duality and hidden symmetries in interacting particle systems. J. Stat. Phys., 135(1):25-55, 2009.

[29] P. Gonçalves. Hydrodynamics for Symmetric Exclusion in Contact with Reservoirs. In G. Giacomin, S. Olla, E. Saada, H. Spohn, and G. Stoltz, editors, Stochastic Dynamics Out of Equilibrium, pages 137-205, Cham, 2019. Springer International Publishing. 
[30] P. Gonçalves, M. Jara, O. Menezes, and A. Neumann. Non-equilibrium and stationary fluctuations for the SSEP with slow boundary. Stochastic Process. Appl., 130(7):4326-4357, 2020.

[31] R. A. Holley and D. W. Stroock. Generalized Ornstein-Uhlenbeck processes and infinite particle branching Brownian motions. Publ. Res. Inst. Math. Sci., 14(3):741-788, 1978.

[32] M. Jara and C. Landim. Quenched non-equilibrium central limit theorem for a tagged particle in the exclusion process with bond disorder. Ann. Inst. Henri Poincaré Probab. Stat., 44(2):341-361, 2008.

[33] W. Jatuviriyapornchai, P. Chleboun, and S. Grosskinsky. Structure of the condensed phase in the inclusion process. J. Stat. Phys., 178(3):682-710, 2020.

[34] G. Kallianpur and J. Xiong. Stochastic differential equations in infinite-dimensional spaces, volume 26 of Institute of Mathematical Statistics Lecture Notes-Monograph Series. Institute of Mathematical Statistics, Hayward, CA, 1995. Expanded version of the lectures delivered as part of the 1993 Barrett Lectures at the University of Tennessee, Knoxville, TN, March 25-27, 1993, With a foreword by Balram S. Rajput and Jan Rosinski.

[35] C. Kipnis and C. Landim. Scaling limits of interacting particle systems, volume 320 of Grundlehren der Mathematischen Wissenschaften. Springer-Verlag, Berlin, 1999.

[36] C. Kipnis, C. Marchioro, and E. Presutti. Heat flow in an exactly solvable model. J. Statist. Phys., 27(1):65-74, 1982.

[37] C. Landim, A. Milanés, and S. Olla. Stationary and nonequilibrium fluctuations in boundary driven exclusion processes. Markov Process. Related Fields, 14(2):165-184, 2008.

[38] C. Landim and K. Tsunoda. Hydrostatics and dynamical large deviations for a reaction-diffusion model. Ann. Inst. Henri Poincaré Probab. Stat., 54(1):51-74, 2018.

[39] T. M. Liggett. Stochastic Interacting Systems: Contact, Voter and Exclusion Processes, volume 324 of Grundlehren der mathematischen Wissenschaften. Springer Berlin Heidelberg, Berlin, Heidelberg, 1999.

[40] T. M. Liggett. Interacting particle systems. Classics in Mathematics. Springer-Verlag, Berlin, 2005. Reprint of the 1985 original.

[41] I. Mitoma. Tightness of probabilities on $\mathcal{C}\left([0,1] ; \mathscr{S}^{\prime}\right)$ and $\mathcal{D}\left([0,1] ; \mathscr{S}^{\prime}\right)$. Ann. Probab., 11(4):989-999, 1983.

[42] Y. Netrusov and Y. Safarov. Weyl asymptotic formula for the Laplacian on domains with rough boundaries. Comm. Math. Phys., 253(2):481-509, 2005.

[43] B. Øksendal. Stochastic differential equations: An introduction with applications. Universitext. Springer-Verlag, Berlin, sixth edition, 2003.

[44] V. Pérez-Abreu and C. Tudor. Regularity and convergence of stochastic convolutions in duals of nuclear Fréchet spaces. J. Multivariate Anal., 43(2):185-199, 1992.

[45] F. Redig and F. Sau. Factorized duality, stationary product measures and generating functions. J. Stat. Phys., 172(4):980-1008, 2018.

[46] C. Stone. Limit theorems for random walks, birth and death processes, and diffusion processes. Illinois J. Math., 7:638-660, 1963.

[47] K. Tsunoda. Hydrostatic limit for exclusion process with slow boundary revisited. In Stochastic Analysis on Large Scale Interacting Systems, RIMS Kôkyûroku Bessatsu, B79, pages 149-162. Res. Inst. Math. Sci. (RIMS), Kyoto, 2020.

[48] K. Vafayi and M. H. Duong. Weakly nonequilibrium properties of a symmetric inclusion process with open boundaries. Phys. Rev. E, 90:052143, 2014.

Center for Mathematical Analysis, Geometry and Dynamical Systems, ist, Universidade de Lisboa, 1049-001 Lisboa, Portugal

Email address: chiara.franceschini@tecnico.ulisboa.pt

Center for Mathematical Analysis, Geometry and Dynamical Systems, ist, Universidade de Lisboa, 1049-001 Lisboa, Portugal

Email address: pgoncalves@tecnico.ulisboa.pt

ist Austria, Am Campus 1, 3400, Klosterneuburg, Austria

Email address: federico.sau@ist.ac.at 